\title{
Identification of an Electrostatic Ruler Motif for Sequence Specific Binding of Collagenase to Collagen
}

\author{
Sundar Raman Subramanian ${ }^{1,2 \#, ~ E . ~ R . ~ A z h a g i y a ~ S i n g a m ~}{ }^{1,2, \#}$ Michael Berinski1 $^{1,3}$, \\ Venkatesan Subramanian², Rebecca C. Wade ${ }^{1,4,5^{*}}$ \\ ${ }^{1}$ Molecular and Cellular Modeling Group, Heidelberg Institute for Theoretical Studies, \\ Schloss-Wolfsbrunnenweg 35, 69117 Heidelberg, Germany \\ ${ }^{2}$ Chemical Laboratory, Central Leather Research Institute, Council of Scientific and \\ Industrial Research, Chennai, India \\ ${ }^{3}$ Edinburgh Genomics. The University of Edinburgh, Scotland, United Kingdom. \\ ${ }^{4}$ Zentrum für Molekulare Biologie der Universität Heidelberg, Heidelberg, Germany \\ ${ }^{5}$ Interdisciplinary Center for Scientific Computing (IWR), Heidelberg University, Germany
}

\footnotetext{
${ }^{\text {\#}}$ Equal first authors

*Correspondence: Tel: +496221533247; Fax:+496221533298; rebecca.wade@h-its.org
}

Running Title: Binding of collagenase to collagen

Keywords: matrix metalloprotease, collagenolysis, computational docking, protein-protein interaction, recognition motif 


\section{Supplemental Methods:}

\section{MD simulation of the model of truncated collagen: setup and equilibration}

MD simulation of the model of truncated collagen was performed using the AMBER 10 package (http://ambermd.org/) with the FF99SB force field ${ }^{1}$ and the parameters of Park et $\mathrm{al}^{2}$ for Hyp. MD simulation was performed employing periodic boundary conditions and an explicit solvent environment with the TIP3P water model ${ }^{3}$ and a minimum of $12.0 \AA$ from the solute to the edge of the solvent box. The Particle Mesh Ewald method was used for calculating the electrostatic interactions and the cutoff for the non-bonded interactions was set to $10 \AA$. The entire system was energy minimized with 1000 cycles of steepest descent followed by 10000 cycles of conjugate gradient minimization. The system was annealed from 0 to $300 \mathrm{~K}$ over $70 \mathrm{ps}$ using a time step of $1 \mathrm{fs}$ with the peptide atoms restrained by a harmonic potential with a force constant of $10 \mathrm{kcal} / \mathrm{mol} / \AA^{2}$. SHAKE was used to constrain bonds involving hydrogen atoms. Water molecules were equilibrated for 50 ps in the NPT ensemble $(1 \mathrm{~atm})$ with the restraints kept on the peptide atoms. The side chains of the truncated collagen were then energy minimized in the solvent equilibrated system with harmonic restraints with a force constant of $1 \mathrm{kcal} / \mathrm{mol} / \AA^{2}$ on the non-hydrogen atoms of the backbone for 100 steps steepest descent followed by 5000 steps conjugate gradient minimization. After this, the entire system was minimized without restraints with the same procedure. The entire system was then equilibrated without restraints for 10 ns in the NPT ensemble at $300 \mathrm{~K}$.

Finally, a production run was performed at $300 \mathrm{~K}$ for $35 \mathrm{~ns}$ in the NPT ensemble. The coordinates of the truncated collagen model were saved at time intervals of $5 \mathrm{~ns}$. These seven structures were minimized with 100 cycles of conjugate gradient energy minimization before using them as the starting geometries for BD docking.

\section{BD rigid body docking protocol}

The SDA program ${ }^{4}$ version 7 , was used to generate the structures of encounter complexes. The docking procedure employs an efficient BD algorithm that mimics the physical process of diffusional association and allows relevant biochemical data to be incorporated as distance constraints. ${ }^{6}$ After docking, the docked configurations are clustered into ensembles that represent potential protein-protein encounter complexes (ECs). This docking procedure has been tested on diverse protein-protein complexes. ${ }^{6}$

All proteins were modelled with a united atom model. In the BD simulations, the proteins were treated as rigid bodies that diffuse with respect to one another under the influence of electrostatic and non-polar forces. ${ }^{6}$ An EC was considered to be formed and therefore recorded when the two proteins satisfied a distance criterion. The distance criterion was chosen such that the distance between any of the $\mathrm{C}_{\beta}$ atoms of the surface residues of collagen and a specified atom in the collagenase (C $\alpha$ (MMP-1) / O (HPX domain only) atom of Ala 280 in the center of the HPX domain, the catalytic Zn ion in the CAT domain) was required to be less than $30 \AA$. The translational and rotational motions were simulated for the collagenase relative to the truncated collagen, which was positioned at the center of the 
simulation space. At the beginning of each trajectory, collagenase was positioned in a random orientation at a random position on the surface of a sphere of radius $b=218 \AA$ centered on the geometric center of collagen. The $b$ radius was chosen to exceed the sum of the maximum radii of both proteins and to be sufficiently large that the forces between the proteins were negligible at this separation. The simulations were performed until the collagenase diffused out of a sphere of radius $c=684 \AA$ centered on collagen.

Trajectories were generated according to the Ermak-McCammon algorithm ${ }^{7}$ with inter-protein electrostatic interaction, electrostatic desolvation and non-polar desolvation forces ${ }^{6 \mathrm{~b}}$ computed as described below. A variable time step was used (minimum value: 0.5 ps), and the relative translational diffusional coefficient was set to $0.0310 \AA \AA^{2} / \mathrm{ps}$, the rotational diffusional coefficient of the MMP-1 or the HPX/CAT domain to $1.79 \mathrm{e}-5 \AA^{2} / \mathrm{ps}$, and the rotational diffusional coefficient of the truncated collagen to $1.73 \mathrm{e}-4 \AA^{2} / \mathrm{ps}$. A temperature of $298 \mathrm{~K}$ and solvent viscosity of $1.002 \mathrm{mPa}$-s were assigned. For each system studied, the simulation of diffusional association was limited to either 2500 trajectories or a total of 500 ns of simulated time.

\section{Computation of inter-protein forces for BD simulations}

Inter-protein electrostatic interaction, electrostatic desolvation and non-polar desolvation forces were computed at each time step of the BD simulations. The electrostatic terms required calculation of the protein electrostatic potentials before starting the BD simulations. All terms required pre-computation of interaction grids so that during the BD simulations, the forces could efficiently be computed from atom-grid interactions.

\section{Calculation of Protein Electrostatic Potentials}

The electrostatic potentials of the proteins were calculated with the Adaptive PoissonBoltzmann Solver (APBS) ${ }^{8}$ program by numerical solution of the linearized finite-difference Poisson-Boltzmann equation. Partial atomic charges and atomic radii were assigned from the optimized potentials for liquid simulations (OPLS) ${ }^{9}$ parameter set, assuming standard protonation states at $\mathrm{pH}$ 7. Parameters developed by Park et al. ${ }^{2}$ were used for Hyp. The charge of the $\mathrm{Ca}^{2+}$ ion was set to +2e. Relative dielectric constants of 1 and 78 were assigned to the interior of the protein and to the solvent, respectively, and the dielectric boundary was defined by the van der Waals surface (smol keyword). Potentials were computed at an ionic strength of $150 \mathrm{mM}$. Grids with a 1.0 A spacing and dimensions of 225 X 225 X 321 and 129 X 129 X 129 points were used to compute the electrostatic potentials of the truncated collagen and the MMP-1 or HPX/CAT domain, respectively. Effective charges for each protein were computed by fitting them so that they reproduce the protein's external potential in a homogeneous dielectric environment corresponding to that of the solvent. ${ }^{10}$ The effective charges were placed on the carboxylate oxygen atoms of Asp and Glu residues and the Cterminus as well as on the amine nitrogen atoms of Lys and Arg residues and the N-terminus.

\section{Calculation of Electrostatic Desolvation Terms}

Electrostatic desolvation grids ${ }^{5}$ were constructed with a spacing of $1.0 \AA$ with the following sizes. The grid dimension was set to 110 X 110 X $410 \AA$ and 110 X 110 X $110 \AA$ for the truncated collagen and the MMP-1 or HPX/CAT domain, respectively. The ionic 
strength and ionic radii were set to $150 \mathrm{mM}$ and $1.5 \AA$. The dielectric constant for the solvent was set to 78. Docking was performed with the edfct $=1$.

\section{Calculation of Non-polar Desolvation Terms}

The non-polar interaction energy is given by an empirical expression dependent on the surface area buried. ${ }^{6 a}$ The non-polar desolvation grids had the same spacing and dimensions as the corresponding electrostatic desolvation grids. The $a, b$ and $\gamma$ parameters were assigned as 3.10, 4.35, and 0.5, respectively, as recommended for protein-protein docking. ${ }^{6 \mathrm{~b}}$ Docking was performed with the hdfct $=-0.013 \mathrm{kcal} / \mathrm{mol}$.

\section{Clustering of BD docking results to define ECs}

For each simulated system, the 1000 configurations saved were clustered using a hierarchical clustering algorithm along with an average linkage rule for the inter-cluster distance calculation $^{6 \mathrm{~b}}$. The distance between the clusters was defined as the average backbone RMSD between docked collagenase structures in one cluster relative to structures in another. Ten clusters ranked by size were generated.

\section{Supporting References}

1. Hornak, V.; Abel, R.; Okur, A.; Strockbine, B.; Roitberg, A.; Simmerling, C., Comparison of multiple Amber force fields and development of improved protein backbone parameters. Proteins 2006, 65, 712-725.

2. $\quad$ Park, S.; Radmer, R. J.; Klein, T. E.; Pande, V. S., A new set of molecular mechanics parameters for hydroxyproline and its use in molecular dynamics simulations of collagen-like peptides. J. Comput. Chem. 2005, 26, 1612-1616.

3. Price, D. J.; Brooks, C. L., 3rd, A modified TIP3P water potential for simulation with Ewald summation. J. Chem. Phys. 2004, 121, 10096-11103.

4. (a) Gabdoulline, R. R.; Wade, R. C., Simulation of the diffusional association of barnase and barstar. Biophys. J. 1997, 72, 1917-1929; (b) Gabdoulline, R. R.; Wade, R. C., Brownian dynamics simulation of protein-protein diffusional encounter. Methods 1998, 14, 329-341; (c) Martinez, M.; Bruce, N. J.; Romanowska, J.; Kokh, D. B.; Ozboyaci, M.; Yu, X. F.; Ozturk, M. A.; Richter, S.; Wade, R. C., SDA 7: A modular and parallel implementation of the simulation of diffusional association software. J. Comput. Chem. 2015, 36, 1631-1645.

5. Elcock, A. H.; Gabdoulline, R. R.; Wade, R. C.; McCammon, J. A., Computer simulation of protein-protein association kinetics: acetylcholinesterase-fasciculin. J. Mol. Biol. 1999, 291, 149-162.

6. (a) Gabdoulline, R. R.; Wade, R. C., On the contributions of diffusion and thermal activation to electron transfer between Phormidium laminosum plastocyanin and cytochrome f: Brownian dynamics simulations with explicit modeling of nonpolar desolvation interactions and electron transfer events. J.. Am. Chem. Soc. 2009, 131, 9230-9238; (b) Motiejunas, D.; Gabdoulline, R.; Wang, T.; Feldman-Salit, A.; Johann, T.; Winn, P. J.; Wade, 
R. C., Protein-protein docking by simulating the process of association subject to biochemical constraints. Proteins 2008, 71, 1955-1969.

7. Ermak, D. L., McCammon J. A., Brownian dynamics with hydrodynamic interactions. J. Chem. Phys. 1978, 69, 1352-1360.

8. Baker, N. A.; Sept, D.; Joseph, S.; Holst, M. J.; McCammon, J. A., Electrostatics of nanosystems: application to microtubules and the ribosome. Proc. Natl. Acad. Sci. USA 2001, 98, 10037-10041.

9. Jorgensen, W. L.; Maxwell, D. S.; Tirado Rives, J., Development and testing of the OPLS all-atom force field on conformational energetics and properties of organic liquids. $J$. Am. Chem. Soc. 1996, 118, 11225-11236.

10. Gabdoulline, R. R.; Wade, R. C., Effective charges for macromolecules in solvent. $J$ Phys. Chem.- 1996, 100, 3868-3878. 
Table S1: Results of the BD rigid body docking of MMP-1 to collagen. Docking was performed for the crystal structure conformation of MMP-1 to seven different conformations of the truncated collagen model generated by MD simulation. The encounter complexes generated in the BD simulations were clustered and ranked according to size. The RMSD was calculated between the structures of MMP-1 present in each cluster. The interaction energy is the sum of electrostatic interaction (ElE), electrostatic desolvation (ElDesE), and nonpolar (np) components. The values for the representative structure in each cluster, as well as the average value of the total interaction energy of the cluster (ClAE) (with standard deviation in brackets), are given. All energies are in $\mathrm{kcal} / \mathrm{mol}$.

\begin{tabular}{|c|c|c|c|c|c|c|}
\hline $\begin{array}{l}\text { Cluster } \\
\text { No. }\end{array}$ & $\begin{array}{c}\text { Cluster Size } \\
(\%)\end{array}$ & $\begin{array}{l}\text { RMSD } \\
(\AA)\end{array}$ & ClAE & $\mathrm{ElE}$ & ElDesE & np \\
\hline \multicolumn{7}{|c|}{ Conformation 1} \\
\hline 1 & 44 & 29.4 & $-20.8(1.0)$ & -4.1 & 4.8 & -20.6 \\
\hline 2 & 21.1 & 22.7 & $-20.7(0.9)$ & -3.9 & 2.2 & -19.5 \\
\hline 3 & 13.8 & 28.2 & $-20.6(0.8)$ & -4.3 & 2.7 & -19.2 \\
\hline 4 & 6.9 & 20.5 & $-20.7(0.8)$ & -2.8 & 1.3 & -18.3 \\
\hline 5 & 5.6 & 17.8 & $-20.3(0.6)$ & -3.5 & 3.4 & -19.6 \\
\hline 6 & 4.8 & 1 & $-20.0(0.2)$ & -2 & 4.7 & -22.7 \\
\hline 7 & 1.8 & 12.5 & $-20.1(0.3)$ & -4 & 2.1 & -18 \\
\hline 8 & 1.7 & 10.7 & $-19.9(0.2)$ & -1.6 & 1.4 & -19.8 \\
\hline 9 & 0.2 & 1 & $-19.8(0.2)$ & -3.7 & 1.9 & -18.3 \\
\hline 10 & 0.2 & 0.6 & $-19.6(0.0)$ & -6.4 & 2.2 & -15.5 \\
\hline \multicolumn{7}{|c|}{ Conformation 2} \\
\hline 1 & 62.8 & 19 & $-21.1(1.0)$ & -3.6 & 1.5 & -17.8 \\
\hline 2 & 12.6 & 6.8 & $-20.5(0.6)$ & -3.2 & 2.6 & -18.9 \\
\hline 3 & 10.7 & 12.7 & $-20.7(1.1)$ & -4.1 & 1.7 & -17.1 \\
\hline 4 & 9.9 & 24.2 & $-20.0(0.6)$ & -5 & 1.8 & -16.2 \\
\hline 5 & 1.2 & 0.9 & $-19.8(0.3)$ & -5.4 & 3.1 & -17.6 \\
\hline 6 & 1.1 & 1.5 & $-20.4(0.6)$ & -4 & 2.4 & -19 \\
\hline 7 & 1.1 & 30.6 & $-19.8(0.5)$ & -5.5 & 2.4 & -16.5 \\
\hline 8 & 0.4 & 13.4 & $-20.0(0.5)$ & -5.2 & 2.6 & -16.8 \\
\hline 9 & 0.1 & 1.5 & $-19.4(0.2)$ & -5.7 & 3.8 & -18.2 \\
\hline 10 & 0 & 0 & $-19.6(0.0)$ & -3.5 & 2 & -18.1 \\
\hline \multicolumn{7}{|c|}{ Conformation 3} \\
\hline 1 & 76.5 & 20.1 & $-21.6(0.8)$ & -1.8 & 2.3 & -20.7 \\
\hline 2 & 7.5 & 11.2 & $-20.7(0.4)$ & -1.1 & 1.3 & -20.8 \\
\hline 3 & 7.1 & 8.7 & $-21.3(0.7)$ & -3.6 & 2.3 & -18.9 \\
\hline 4 & 5.2 & 19.6 & $-20.9(0.4)$ & -3.6 & 1.6 & -18.1 \\
\hline 5 & 1.4 & 1.2 & $-20.9(0.3)$ & -2.5 & 2.2 & -20.7 \\
\hline 6 & 0.8 & 1.2 & $-20.9(0.5)$ & -5.4 & 2.3 & -17.6 \\
\hline 7 & 0.8 & 3.8 & $-20.4(0.3)$ & -2.3 & 1.5 & -19.3 \\
\hline 8 & 0.4 & 18.9 & $-20.1(0.0)$ & -3.6 & 2.1 & -18.6 \\
\hline
\end{tabular}




\begin{tabular}{|c|c|c|c|c|c|c|}
\hline 9 & 0.2 & 1.2 & $-20.3(0.3)$ & -4.9 & 7.9 & -23.3 \\
\hline 10 & 0.1 & 0 & $-20.2(0.0)$ & -2.4 & 3.6 & -21.5 \\
\hline \multicolumn{7}{|c|}{ Conformation 4} \\
\hline 1 & 75.1 & 15.8 & $-21.6(1.8)$ & -5 & 1.9 & -19.3 \\
\hline 2 & 9.2 & 25.1 & $-19.9(0.4)$ & -4.4 & 2.6 & -18 \\
\hline 3 & 8.9 & 8.9 & $-20.1(0.5)$ & -2 & 1 & -18.5 \\
\hline 4 & 3.8 & 14 & $-20.0(0.6)$ & -3.8 & 2.9 & -20.2 \\
\hline 5 & 1.2 & 1.2 & $-19.8(0.4)$ & -3 & 1.5 & -18.2 \\
\hline 6 & 1.2 & 1.4 & $-19.3(0.0)$ & -5.8 & 2.7 & -16.3 \\
\hline 7 & 0.5 & 10.7 & $-19.3(0.1)$ & -2.8 & 4.5 & -20.9 \\
\hline 8 & 0.1 & 0.5 & $-19.9(0.3)$ & -3.4 & 3.1 & -20 \\
\hline 9 & 0 & 0 & $-19.3(0.0)$ & -3.4 & 3.8 & -19.7 \\
\hline 10 & 0 & 0 & $-19.4(0.0)$ & -4.4 & 3.7 & -18.7 \\
\hline \multicolumn{7}{|c|}{ Conformation 5} \\
\hline 1 & 30.1 & 9.3 & $-21.9(1.3)$ & -3.4 & 2.4 & -21.5 \\
\hline 2 & 24.6 & 12.5 & $-21.0(1.1)$ & -3.7 & 2.6 & -18.2 \\
\hline 3 & 23.6 & 27.5 & $-21.0(1.0)$ & -1.6 & 2.4 & -20.3 \\
\hline 4 & 9.7 & 11.3 & $-22.0(1.4)$ & -0.8 & 2.2 & -20.8 \\
\hline 5 & 8.3 & 10.4 & $-22.1(1.6)$ & -0.1 & 4 & -23.4 \\
\hline 6 & 2.4 & 15.8 & $-19.5(0.3)$ & -5.2 & 2.4 & -16.4 \\
\hline 7 & 1 & 2.9 & $-19.6(0.4)$ & -2.5 & 2.3 & -19 \\
\hline 8 & 0.2 & 1.5 & $-19.7(0.2)$ & -1.6 & 2.8 & -20.8 \\
\hline 9 & 0.2 & 0.4 & $-19.4(0.1)$ & -5.2 & 4 & -18.4 \\
\hline 10 & 0 & 0 & $-19.6(0.0)$ & -3.7 & 2.2 & -18 \\
\hline \multicolumn{7}{|c|}{ Conformation 6} \\
\hline 1 & 38 & 19.3 & $-20.8(1.2)$ & -3.7 & 1.6 & -17.5 \\
\hline 2 & 23.4 & 15 & $-20.3(0.8)$ & -6.4 & 2.5 & -15.7 \\
\hline 3 & 16.6 & 17.9 & $-20.5(0.9)$ & -3.8 & 2 & -17.7 \\
\hline 4 & 9 & 9.3 & $-19.9(0.4)$ & -5.6 & 6 & -19.8 \\
\hline 5 & 7.5 & 23.7 & $-19.9(0.5)$ & -2.7 & 1.3 & -18 \\
\hline 6 & 2.7 & 7.3 & $-20.6(0.7)$ & -3.8 & 2.3 & -18.6 \\
\hline 7 & 1.4 & 5.8 & $-19.8(0.3)$ & -3.2 & 1.9 & -18.2 \\
\hline 8 & 1 & 0.9 & $-19.9(0.5)$ & -6.7 & 4.6 & -18.6 \\
\hline 9 & 0.3 & 16.8 & $-19.4(0.1)$ & -3.3 & 4.2 & -20.2 \\
\hline 10 & 0.1 & 2.3 & $-19.5(0.2)$ & -2.9 & 3.3 & -20.2 \\
\hline \multicolumn{7}{|c|}{ Conformation 7} \\
\hline 1 & 44.5 & 16.5 & $-21.3(1.0)$ & -1.2 & 3 & -25.7 \\
\hline 2 & 29.4 & 8.7 & $-21.8(1.6)$ & -2.8 & 2.3 & -19.6 \\
\hline 3 & 5 & 2.6 & $-20.0(0.4)$ & -4.2 & 2.2 & -17.8 \\
\hline 4 & 4.9 & 8 & $-21.2(1.0)$ & -2.1 & 2.8 & -20.5 \\
\hline 5 & 4.6 & 1 & $-20.8(0.6)$ & -6.5 & 6.8 & -21.5 \\
\hline 6 & 4.1 & 1.6 & $-20.5(0.5)$ & -4.1 & 3.3 & -19.8 \\
\hline 7 & 3.2 & 10.2 & $-20.7(0.7)$ & -3.6 & 2.4 & -18.9 \\
\hline
\end{tabular}




\begin{tabular}{ccccccc}
8 & 2.4 & 3.3 & $-19.9(0.3)$ & -3.6 & 2.9 & -19.4 \\
9 & 1.5 & 24.2 & $-19.9(0.3)$ & -2.5 & 1.2 & -18.6 \\
10 & 0.3 & 0.3 & $-19.8(0.2)$ & 0.1 & 1.5 & -21.6 \\
\hline
\end{tabular}


Table S2: Conformations of the side chains of Arg residues in the seven truncated collagen models.

\begin{tabular}{|c|c|c|c|c|c|c|}
\hline \multirow[t]{2}{*}{ Subsite } & \multirow[t]{2}{*}{ Chain } & \multirow[t]{2}{*}{ Model } & \multicolumn{4}{|c|}{ Dihedral angle $\left(^{\circ}\right)$} \\
\hline & & & c1 & c2 & c3 & c4 \\
\hline \multirow[t]{21}{*}{ P8’ } & \multirow[t]{7}{*}{ A } & 1 & -173 & 175 & -174 & -174 \\
\hline & & 2 & -167 & 175 & -160 & 163 \\
\hline & & 3 & -174 & 79 & -161 & -95 \\
\hline & & 4 & -175 & 172 & -63 & -86 \\
\hline & & 5 & -165 & 169 & -163 & 169 \\
\hline & & 6 & -175 & -175 & 67 & -170 \\
\hline & & 7 & -173 & -170 & 69 & -169 \\
\hline & \multirow[t]{7}{*}{ B } & 1 & 177 & 69 & 76 & 163 \\
\hline & & 2 & -170 & 176 & -167 & -168 \\
\hline & & 3 & -176 & 176 & -169 & -178 \\
\hline & & 4 & 87 & -61 & -173 & 132 \\
\hline & & 5 & -174 & -171 & -82 & -175 \\
\hline & & 6 & -157 & -166 & -175 & 170 \\
\hline & & 7 & -173 & 171 & -164 & 174 \\
\hline & \multirow[t]{7}{*}{$\mathrm{C}$} & 1 & -166 & 177 & -179 & -161 \\
\hline & & 2 & -167 & -179 & -177 & -173 \\
\hline & & 3 & -170 & 177 & -170 & -170 \\
\hline & & 4 & -171 & 71 & 180 & -71 \\
\hline & & 5 & -166 & 178 & 179 & -169 \\
\hline & & 6 & -170 & 171 & -173 & -149 \\
\hline & & 7 & -157 & -175 & 180 & -174 \\
\hline \multirow[t]{17}{*}{ P20’ } & \multirow[t]{7}{*}{ A } & 1 & -171 & 174 & -177 & -88 \\
\hline & & 2 & -167 & -177 & -176 & 76 \\
\hline & & 3 & -164 & -166 & -163 & -173 \\
\hline & & 4 & -175 & 177 & -156 & -179 \\
\hline & & 5 & -170 & 163 & -161 & -163 \\
\hline & & 6 & -175 & 169 & 174 & -79 \\
\hline & & 7 & -171 & 170 & -60 & -79 \\
\hline & \multirow[t]{7}{*}{ B } & 1 & -176 & 172 & 60 & -116 \\
\hline & & 2 & -180 & 175 & -174 & -115 \\
\hline & & 3 & -170 & 173 & 179 & -128 \\
\hline & & 4 & -177 & -176 & 176 & 75 \\
\hline & & 5 & -167 & -178 & -175 & -168 \\
\hline & & 6 & 73 & -177 & -73 & 103 \\
\hline & & 7 & -169 & 160 & -177 & -133 \\
\hline & \multirow[t]{3}{*}{ C } & 1 & -162 & -178 & -178 & 176 \\
\hline & & 2 & -167 & -174 & -176 & -171 \\
\hline & & 3 & -158 & -175 & -178 & -163 \\
\hline
\end{tabular}




\begin{tabular}{|c|c|c|c|c|c|c|}
\hline & & 4 & -163 & -177 & -165 & 170 \\
\hline & & 5 & -161 & -171 & -170 & 172 \\
\hline & & 6 & -160 & -178 & -180 & -180 \\
\hline & & 7 & -167 & 174 & -172 & -162 \\
\hline \multirow[t]{21}{*}{ P44' } & \multirow[t]{7}{*}{ A } & 1 & -178 & 173 & 66 & -171 \\
\hline & & 2 & -175 & 168 & 67 & -178 \\
\hline & & 3 & 175 & 175 & 56 & 170 \\
\hline & & 4 & -179 & 164 & 70 & -165 \\
\hline & & 5 & 172 & 178 & -86 & 156 \\
\hline & & 6 & -169 & 171 & -70 & 114 \\
\hline & & 7 & -171 & 161 & -169 & -142 \\
\hline & \multirow[t]{7}{*}{ B } & 1 & -169 & 177 & -61 & 123 \\
\hline & & 2 & -57 & -169 & 69 & 112 \\
\hline & & 3 & -171 & 160 & 179 & -143 \\
\hline & & 4 & -169 & 168 & -164 & -160 \\
\hline & & 5 & -178 & 172 & -159 & -139 \\
\hline & & 6 & -179 & -175 & -68 & -80 \\
\hline & & 7 & -168 & -171 & 69 & 172 \\
\hline & \multirow[t]{7}{*}{$\mathrm{C}$} & 1 & -170 & 172 & 73 & -136 \\
\hline & & 2 & -73 & -180 & 180 & -83 \\
\hline & & 3 & -170 & 174 & -66 & -86 \\
\hline & & 4 & -168 & 173 & -66 & -96 \\
\hline & & 5 & -176 & 178 & 59 & -172 \\
\hline & & 6 & 74 & 166 & -180 & 101 \\
\hline & & 7 & -164 & 173 & -167 & -155 \\
\hline
\end{tabular}


Table S3: Interfacial residues between collagen and MMP-1. The closest contact distances are given for residues with non-hydrogen atoms less than $4.5 \AA$ apart making contacts between collagen and MMP-1 in the most populated representative encounter complexes obtained from SDA simulations for conformations 2-7 of collagen in which the HPX domain binds between the arginines of the electrostatic motif of collagen.

\begin{tabular}{|c|c|c|c|c|c|c|c|c|c|c|c|c|c|}
\hline $\begin{array}{l}\text { Collagen } \\
\text { Conform- } \\
\text { ation No. }\end{array}$ & $\begin{array}{l}\text { Collagen } \\
\text { (Res) }\end{array}$ & $\begin{array}{l}\text { Residue } \\
\text { No }\end{array}$ & $\begin{array}{l}\text { Chain } \\
\text { ID }\end{array}$ & $\begin{array}{l}\text { CAT } \\
\text { Domain } \\
\text { (Res) }\end{array}$ & $\begin{array}{l}\text { Res } \\
\text { No }\end{array}$ & $\begin{array}{l}\text { Distance } \\
\text { (A)) }\end{array}$ & $\begin{array}{l}\text { Collagen } \\
\text { Conform- } \\
\text { ation No. }\end{array}$ & $\begin{array}{l}\text { Collagen } \\
\text { (Res) }\end{array}$ & $\begin{array}{l}\text { Residue } \\
\text { No }\end{array}$ & $\begin{array}{l}\text { Chain } \\
\text { ID }\end{array}$ & $\begin{array}{l}\text { HPX } \\
\text { Domain } \\
\text { (Res) }\end{array}$ & $\begin{array}{l}\text { Residue } \\
\text { No }\end{array}$ & $\begin{array}{l}\text { Distance } \\
\text { (Å) }\end{array}$ \\
\hline 2 & HYP & P5 & $\mathrm{C}$ & ASP & 167 & 4.36 & 2 & MET & P16' & $\mathrm{C}$ & GLY & 292 & 4.43 \\
\hline 2 & LEU & P2 & C & PRO & 173 & 4.31 & 2 & MET & P16’ & B & ASP & 338 & 3.55 \\
\hline 2 & LEU & P2 & $\mathrm{C}$ & PHE & 174 & 4.30 & & & & & & & \\
\hline 2 & ILE & P4' & A & ASP & 175 & 3.99 & & & & & & & \\
\hline 2 & LEU & P2 & $\mathrm{C}$ & ASP & 175 & 4.30 & & & & & & & \\
\hline 3 & THR & P5' & $\mathrm{C}$ & ASN & 171 & 3.87 & 3 & PRO & P19' & $\mathrm{C}$ & ARG & 291 & 4.22 \\
\hline 3 & ILE & P4' & $\mathrm{C}$ & ASN & 171 & 4.46 & 3 & MET & P16' & $\mathrm{C}$ & TYR & 309 & 4.16 \\
\hline 3 & THR & P5' & $\mathrm{C}$ & SER & 172 & 3.75 & 3 & PRO & P19' & C & GLN & 354 & 4.43 \\
\hline 3 & LEU & P10' & A & ASN & 180 & 4.20 & & & & & & & \\
\hline 3 & PRO & P13' & B & ASN & 239 & 3.22 & & & & & & & \\
\hline 4 & ILE & P1' & B & ASN & 171 & 3.84 & 4 & HYP & P17’ & A & ARG & 291 & 4.23 \\
\hline 4 & LEU & P2 & B & GLN & 186 & 4.23 & 4 & HYP & P14' & A & PHE & 308 & 4.37 \\
\hline 4 & ILE & P4 & $\mathrm{C}$ & $\mathrm{HIG}$ & 228 & 4.47 & 4 & HYP & P14' & A & TYR & 309 & 4.37 \\
\hline 4 & ILE & P4 & $\mathrm{C}$ & PRO & 238 & 4.42 & 4 & ARG & P8' & $\mathrm{C}$ & GLU & 313 & 3.72 \\
\hline 5 & ILE & P4' & B & GLY & 166 & 4.35 & 5 & PRO & P19' & A & ARG & 291 & 4.08 \\
\hline 5 & ILE & P4' & B & ASP & 167 & 4.37 & 5 & MET & P16' & A & ARG & 304 & 3.70 \\
\hline \multirow[t]{4}{*}{5} & THR & P5' & B & ASP & 175 & 4.07 & 5 & ARG & P20' & A & SER & 307 & 4.33 \\
\hline & & & & & & & 5 & MET & P16' & A & TYR & 309 & 3.62 \\
\hline & & & & & & & 5 & HYP & P23' & A & ALA & 335 & 4.15 \\
\hline & & & & & & & 5 & PRO & P25' & B & GLU & 384 & 4.44 \\
\hline 6 & ILE & P1' & B & SER & 172 & 4.30 & 6 & MET & P16' & A & ARG & 291 & 3.82 \\
\hline 6 & ILE & P1' & B & GLN & 186 & 4.17 & 6 & HYP & P14' & A & PHE & 308 & 4.17 \\
\hline 6 & THR & P5' & B & PRO & 238 & 3.86 & & & & & & & \\
\hline 7 & ILE & P4' & $\mathrm{C}$ & PRO & 104 & 4.21 & 7 & ARG & P20' & A & ARG & 291 & 4.31 \\
\hline
\end{tabular}




\begin{tabular}{|c|c|c|c|c|c|c|c|c|c|c|c|c|c|}
\hline 7 & ILE & P4' & B & ASN & 171 & 4.21 & 7 & HYP & P17' & A & ARG & 304 & 3.67 \\
\hline 7 & ARG & P8' & B & ASN & 180 & 4.29 & 7 & MET & P16' & B & TYR & 309 & 4.40 \\
\hline 7 & PRO & P13’ & A & TYR & 237 & 4.14 & 7 & HYP & P17’ & A & GLU & 313 & 3.56 \\
\hline 7 & PRO & P13’ & A & ASN & 239 & 3.57 & 7 & MET & P16' & A & VAL & 319 & 4.27 \\
\hline 7 & ALA & P11' & $\mathrm{C}$ & ASN & 239 & 4.09 & & & & & & & \\
\hline 7 & GLY & P9' & C & ASN & 239 & 4.39 & & & & & & & \\
\hline
\end{tabular}


Table S4: Results of the BD rigid body docking of the HPX domain of collagenase to collagen. Docking was performed for seven different conformations of the truncated collagen model generated by MD simulation. The encounter complexes generated in the BD simulations were clustered and ranked according to size. The RMSD was calculated between the structures of HPX present in each cluster. The interaction energy is the sum of electrostatic interaction (ElE), electrostatic desolvation (ElDesE) and nonpolar (np) components. The values for the representative structure in each cluster, as well as the average value of the total interaction energy of the cluster (ClAE) (with standard deviation in brackets), are given. All energies are in $\mathrm{kcal} / \mathrm{mol}$.

\begin{tabular}{|c|c|c|c|c|c|c|}
\hline $\begin{array}{l}\text { Cluster } \\
\text { No. }\end{array}$ & $\begin{array}{c}\text { Cluster Size } \\
(\%)\end{array}$ & $\begin{array}{c}\text { RMSD } \\
(\AA)\end{array}$ & ClAE & ElE & ElDesE & np \\
\hline \multicolumn{7}{|c|}{ Conformation 1} \\
\hline 1 & 36.9 & 6.2 & $-20.7(1.0)$ & -2.3 & 5.7 & -23.7 \\
\hline 2 & 16.5 & 14 & $-19.7(0.7)$ & -4.6 & 2.2 & -16.7 \\
\hline 3 & 12.6 & 8.4 & $-19.3(0.5)$ & -5.2 & 2.4 & -15.9 \\
\hline 4 & 11.6 & 12.5 & $-19.5(0.7)$ & -6 & 3 & -15.8 \\
\hline 5 & 8.7 & 9.4 & $-19.2(0.5)$ & -5.9 & 3.3 & -16.9 \\
\hline 6 & 6.1 & 15.7 & $-19.3(0.7)$ & -5.2 & 2.6 & -16.1 \\
\hline 7 & 4.2 & 14.1 & $-19.0(0.3)$ & -5.7 & 3.1 & -16.9 \\
\hline 8 & 1.8 & 5.4 & $-19.0(0.3)$ & -6.9 & 3.5 & -15.9 \\
\hline 9 & 1.4 & 7.3 & $-19.0(0.3)$ & -3.3 & 1.6 & -16.9 \\
\hline 10 & 0.3 & 15.8 & $-18.7(0.1)$ & -6.3 & 3.7 & -16.2 \\
\hline \multicolumn{7}{|c|}{ Conformation 2} \\
\hline 1 & 26 & 10.2 & $-20.8(0.5)$ & -7 & 3.1 & -16.6 \\
\hline 2 & 25.9 & 3.5 & $-21.2(0.7)$ & -5.4 & 3.8 & -18.6 \\
\hline 3 & 19.6 & 17 & $-20.6(0.6)$ & -5.6 & 2 & -16.5 \\
\hline 4 & 10.4 & 4.8 & $-20.8(0.6)$ & -5.9 & 3.7 & -17.9 \\
\hline 5 & 9.6 & 7.8 & $-20.8(0.6)$ & -5 & 2.7 & -18.7 \\
\hline 6 & 6.1 & 6.9 & $-20.8(0.6)$ & -6.1 & 3.2 & -17 \\
\hline 7 & 1.3 & 9 & $-20.2(0.3)$ & -6.2 & 2.5 & -16.2 \\
\hline 8 & 0.8 & 0.5 & $-20.2(0.1)$ & -2.3 & 1.8 & -19.8 \\
\hline 9 & 0.3 & 0.6 & $-20.3(0.3)$ & -5 & 2.2 & -17.7 \\
\hline 10 & 0 & 0 & $-19.9(0.0)$ & -5.9 & 3.9 & -18 \\
\hline \multicolumn{7}{|c|}{ Conformation 3} \\
\hline 1 & 50.9 & 11.6 & $-21.3(1.1)$ & -5.1 & 1.9 & -17.8 \\
\hline 2 & 25.1 & 9.6 & $-21.7(0.8)$ & -4.5 & 1.3 & -17.4 \\
\hline 3 & 11 & 9.3 & $-20.9(0.7)$ & -3.3 & 3.1 & -20.2 \\
\hline 4 & 6.5 & 4 & $-20.7(0.5)$ & -4.8 & 3.1 & -19 \\
\hline 5 & 3.8 & 1 & $-20.7(0.4)$ & -9.1 & 8.1 & -19.8 \\
\hline 6 & 1.2 & 5.5 & $-20.3(0.2)$ & -3.9 & 2.7 & -19.2 \\
\hline 7 & 0.9 & 3 & $-20.3(0.1)$ & -4 & 2.7 & -18.8 \\
\hline 8 & 0.4 & 0.9 & $-20.5(0.2)$ & -5.8 & 5.9 & -20.6 \\
\hline 9 & 0.1 & 0.8 & $-20.3(0.3)$ & -5 & 2.9 & -18.9 \\
\hline
\end{tabular}




\begin{tabular}{|c|c|c|c|c|c|c|}
\hline 10 & 0.1 & 0 & $-20.2(0.0)$ & -3.4 & 2.5 & -19.4 \\
\hline \multicolumn{7}{|c|}{ Conformation 4} \\
\hline 1 & 34.1 & 11.9 & $-20.2(0.8)$ & -5.2 & 3 & -18.5 \\
\hline 2 & 23.2 & 18.9 & $-19.8(0.5)$ & -7.7 & 4.3 & -15.9 \\
\hline 3 & 18.2 & 7.5 & $-19.7(0.4)$ & -5.4 & 2.7 & -16.7 \\
\hline 4 & 10.6 & 15.5 & $-20.2(1.0)$ & -4.7 & 2.3 & -17.2 \\
\hline 5 & 4.2 & 13.6 & $-19.5(0.3)$ & -5.1 & 2.2 & -16.4 \\
\hline 6 & 3.7 & 4.8 & $-19.4(0.2)$ & -2.9 & 3.4 & -19.9 \\
\hline 7 & 3.2 & 0.6 & $-19.4(0.2)$ & -1.8 & 2.6 & -20.3 \\
\hline 8 & 1.9 & 5.4 & $-19.7(0.4)$ & -3.1 & 2.3 & -18.3 \\
\hline 9 & 0.6 & 0.9 & $-19.7(0.5)$ & -6.1 & 5 & -18.7 \\
\hline 10 & 0.4 & 0.3 & $-20.1(0.2)$ & -4 & 3 & -19.3 \\
\hline \multicolumn{7}{|c|}{ Conformation 5} \\
\hline 1 & 43.2 & 13.6 & $-21.9(1.7)$ & -6 & 2.6 & -16 \\
\hline 2 & 21.9 & 9 & $-19.6(0.8)$ & -5.4 & 2.4 & -15.8 \\
\hline 3 & 7.6 & 7.1 & $-19.9(0.7)$ & -4.8 & 2.1 & -18 \\
\hline 4 & 7.4 & 3.4 & $-20.8(1.0)$ & -5.4 & 2.5 & -16 \\
\hline 5 & 6.8 & 13.5 & $-19.2(0.5)$ & -6.2 & 3 & -15.5 \\
\hline 6 & 6.1 & 28 & $-19.3(0.8)$ & -4.5 & 3.9 & -19 \\
\hline 7 & 5.9 & 21.7 & $-19.0(0.4)$ & -5.2 & 3.6 & -17.3 \\
\hline 8 & 0.8 & 18.4 & $-18.9(0.3)$ & -4.3 & 2.7 & -16.8 \\
\hline 9 & 0.1 & 0.5 & $-19.1(0.1)$ & -5.3 & 4.8 & -18.7 \\
\hline 10 & 0.1 & 0 & $-18.7(0.0)$ & -0.2 & 1.5 & -20 \\
\hline \multicolumn{7}{|c|}{ Conformation 6} \\
\hline 1 & 38.7 & 13.2 & $-21.3(1.7)$ & -6.4 & 3.7 & -18 \\
\hline 2 & 19.5 & 9.4 & $-19.9(0.7)$ & -8 & 3 & -14.3 \\
\hline 3 & 10.4 & 1.6 & $-21.0(1.0)$ & -5 & 2.1 & -19.1 \\
\hline 4 & 9.2 & 17.6 & $-19.2(0.4)$ & -6.9 & 3.6 & -15.8 \\
\hline 5 & 7.9 & 6.9 & $-19.8(0.6)$ & -2.3 & 2 & -18.8 \\
\hline 6 & 6.7 & 7.1 & $-19.2(0.3)$ & -8 & 6.1 & -16.9 \\
\hline 7 & 3.7 & 6.7 & $-19.4(0.4)$ & -3.7 & 3.6 & -19 \\
\hline 8 & 1.5 & 4.3 & $-19.3(0.3)$ & -4.3 & 4.3 & -19.5 \\
\hline 9 & 1.2 & 1 & $-19.8(0.5)$ & -4.7 & 3.6 & -19.1 \\
\hline 10 & 1.1 & 1 & $-19.2(0.2)$ & -2.3 & 2.4 & -19.6 \\
\hline \multicolumn{7}{|c|}{ Conformation 7} \\
\hline 1 & 46.9 & 3.1 & $-20.3(0.6)$ & -4.9 & 3.4 & -19.7 \\
\hline 2 & 20.4 & 15.9 & $-19.4(0.7)$ & -7.1 & 3.6 & -16.2 \\
\hline 3 & 12.4 & 12.5 & $-20.2(1.1)$ & -4.3 & 2 & -17 \\
\hline 4 & 7.3 & 18 & $-19.6(0.8)$ & -5.7 & 3.6 & -16.6 \\
\hline 5 & 5.2 & 1.6 & $-19.2(0.3)$ & -3.4 & 3 & -18.2 \\
\hline 6 & 3.3 & 10.6 & $-19.1(0.5)$ & -6.6 & 3.2 & -15.5 \\
\hline 7 & 3 & 2.6 & $-19.3(0.5)$ & -3.7 & 3.4 & -19.3 \\
\hline 8 & 1.1 & 20.3 & $-19.5(0.6)$ & -5.1 & 3.2 & -16.8 \\
\hline
\end{tabular}




\begin{tabular}{ccccccc}
9 & 0.2 & 4.3 & $-18.8(0.1)$ & -4.5 & 3.3 & -17.5 \\
10 & 0.2 & 1.3 & $-18.8(0.1)$ & -5.3 & 3.5 & -17 \\
\hline
\end{tabular}


Table S5: Results of the BD rigid body docking of the CAT domain of collagenase to collagen. Docking was performed for seven different conformations of the truncated collagen model generated by MD simulation. The encounter complexes generated in the BD simulations were clustered and ranked according to size. The RMSD was calculated between the structures of the CAT domain present in each cluster. The interaction energy is the sum of electrostatic interaction (ElE), electrostatic desolvation (ElDesE), and nonpolar (np) components. The values for the representative structure in each cluster, as well as the average value of the total interaction energy in the cluster (ClAE) (with standard deviation in brackets), are given. All energies are in $\mathrm{kcal} / \mathrm{mol}$.

\begin{tabular}{|c|c|c|c|c|c|c|}
\hline Cluster No. & Cluster Size (\%) & RMSD $(\AA ̊)$ & $\mathrm{ClAE}$ & ElE & ElDesE & np \\
\hline \multicolumn{7}{|c|}{ Conformation -1} \\
\hline 1 & 34.8 & 22.4 & $-16.3(1.1)$ & -3.4 & 1.2 & -13.5 \\
\hline 2 & 22.4 & 16.4 & $-16.0(0.8)$ & -5.2 & 2.6 & -14 \\
\hline 3 & 14.2 & 6.6 & $-16.2(0.7)$ & -2 & 1.1 & -15.9 \\
\hline 4 & 11.9 & 10.9 & $-15.9(0.5)$ & -0.8 & 0.9 & -15.2 \\
\hline 5 & 8.4 & 6.9 & $-16.1(0.9)$ & -1.5 & 2.3 & -17.5 \\
\hline 6 & 3.4 & 3.4 & $-15.9(0.4)$ & -3.2 & 2.1 & -14.9 \\
\hline 7 & 2.3 & 19.9 & $-15.4(0.2)$ & 0.1 & 0.4 & -15.5 \\
\hline 8 & 2.1 & 11.3 & $-15.2(0.4)$ & -1.2 & 1.4 & -15.4 \\
\hline 9 & 0.4 & 1.1 & $-15.3(0.3)$ & 0.1 & 1 & -16.1 \\
\hline 10 & 0 & 0 & $-15.0(0.4)$ & -3.3 & 3.2 & -14.8 \\
\hline \multicolumn{7}{|c|}{ Conformation -2} \\
\hline 1 & 29.3 & 21.3 & $-16.1(0.7)$ & -1.3 & 0.9 & -15.5 \\
\hline 2 & 28.6 & 7.7 & $-17.4(1.1)$ & -3.1 & 1.7 & -13.9 \\
\hline 3 & 12.3 & 15.4 & $-16.7(0.9)$ & -2.3 & 1.8 & -17 \\
\hline 4 & 9 & 16.5 & $-16.1(0.7)$ & -2.9 & 0.9 & -14.2 \\
\hline 5 & 6.4 & 16 & $-16.00 .6)$ & -5.4 & 2.5 & -13.2 \\
\hline 6 & 6 & 8.4 & $-16.1(0.7)$ & -2.2 & 1.6 & -15.4 \\
\hline 7 & 3.6 & 8.5 & $-16.1(0.2)$ & -4.4 & 2.5 & -14.4 \\
\hline 8 & 2.9 & 22.2 & $-16.0(0.3)$ & 0.3 & 0.8 & -16.9 \\
\hline 9 & 1.5 & 12.7 & $-15.9(0.4)$ & -1.9 & 2.1 & -17 \\
\hline 10 & 0.4 & 5.4 & $-15.5(0.0)$ & -0.1 & 0.9 & -16.3 \\
\hline \multicolumn{7}{|c|}{ Conformation -3} \\
\hline 1 & 48.7 & 5.5 & $-18.1(0.9)$ & -3.1 & 2 & -14.8 \\
\hline 2 & 17.2 & 23.8 & $-17.0(0.7)$ & -0.2 & 1.3 & -16.9 \\
\hline 3 & 11 & 17.2 & $-16.5(0.8)$ & -1 & 1 & -15.9 \\
\hline 4 & 6.4 & 10.7 & $-16.2(0.4)$ & -0.2 & 0.7 & -16.3 \\
\hline 5 & 5.6 & 18 & $-16.2(0.7)$ & -2.8 & 1.1 & -14.1 \\
\hline 6 & 3.2 & 8.9 & $-15.8(0.5)$ & 0.2 & 0.8 & -16.8 \\
\hline 7 & 2.8 & 15.2 & $-16.2(0.4)$ & -3.9 & 3.2 & -15 \\
\hline 8 & 2.7 & 7.5 & $-16.7(0.2)$ & -0.4 & 1.1 & -17.6 \\
\hline 9 & 1.9 & 2.8 & $-15.7(0.3)$ & -0.3 & 1.1 & -16.5 \\
\hline
\end{tabular}




\begin{tabular}{|c|c|c|c|c|c|c|}
\hline 10 & 0.5 & 1.3 & $-15.7(0.0)$ & 0.1 & 0.7 & -16.5 \\
\hline \multicolumn{7}{|c|}{ Conformation -4 } \\
\hline 1 & 36.8 & 14.5 & $-16.1(1.2)$ & -1.2 & 1 & -14.5 \\
\hline 2 & 17.6 & 14.6 & $-15.6(0.6)$ & -2.3 & 1.3 & -15.3 \\
\hline 3 & 13.2 & 24.1 & $-15.1(0.6)$ & -0.9 & 0.7 & -14.3 \\
\hline 4 & 11.3 & 22.3 & $-15.3(0.7)$ & -2 & 2.5 & -15 \\
\hline 5 & 8.7 & 28 & $-15.5(0.6)$ & -3 & 2.8 & -14.4 \\
\hline 6 & 5.4 & 9 & $-15.2(0.9)$ & -2.5 & 2.5 & -15.1 \\
\hline 7 & 3 & 1.9 & $-15.2(0.5)$ & -2.9 & 2.7 & -15.3 \\
\hline 8 & 2.9 & 23.3 & $-14.9(0.5)$ & -0.2 & 0.7 & -15.2 \\
\hline 9 & 1.1 & 2.1 & $-15.2(0.5)$ & -2.1 & 0.8 & -14.4 \\
\hline 10 & 0 & 0 & $-14.6(0.0)$ & 0 & 2 & -16.5 \\
\hline \multicolumn{7}{|c|}{ Conformation -5} \\
\hline 1 & 28.6 & 17.5 & $-15.3(0.9)$ & -0.8 & 1.1 & -15.8 \\
\hline 2 & 27.1 & 25 & $-15.2(0.5)$ & -3.5 & 2.6 & -13.4 \\
\hline 3 & 21.5 & 21.2 & $-15.3(1.3)$ & -3.4 & 2 & -13.1 \\
\hline 4 & 11.2 & 24.9 & $-15.0(0.9)$ & -0.2 & 1 & -16.7 \\
\hline 5 & 9.4 & 17.5 & $-14.8(0.9)$ & -2.1 & 1 & -13.9 \\
\hline 6 & 0.6 & 5.1 & $-14.4(0.7)$ & -2.4 & 3.4 & -15.5 \\
\hline 7 & 0.6 & 1.1 & $-14.4(0.5)$ & -2.5 & 2.2 & -13.9 \\
\hline 8 & 0.4 & 20.7 & $-14.4(0.4)$ & -1 & 1.2 & -14.9 \\
\hline 9 & 0.4 & 17 & $-14.4(0.2)$ & 0.1 & 0.7 & -15 \\
\hline 10 & 0.1 & 0 & $-14.5(0.1)$ & -0.1 & 0.5 & -14.8 \\
\hline \multicolumn{7}{|c|}{ Conformation -6} \\
\hline 1 & 34.9 & 17.8 & $-15.3(0.8)$ & -2.3 & 1.2 & -14 \\
\hline 2 & 30.1 & 21 & $-14.9(0.7)$ & -0.7 & 1 & -14.9 \\
\hline 3 & 10 & 21 & $-15.0(0.9)$ & 0.5 & 2.8 & -18.2 \\
\hline 4 & 8.9 & 12.9 & $-14.9(0.6)$ & -2.3 & 0.8 & -13 \\
\hline 5 & 6.1 & 12.8 & $-15.2(0.7)$ & 0 & 0.8 & -15.3 \\
\hline 6 & 3.7 & 10.5 & $-14.5(1.1)$ & -0.5 & 1.3 & -15.3 \\
\hline 7 & 3.7 & 5.9 & $-14.5(0.4)$ & -0.1 & 1 & -15.2 \\
\hline 8 & 1.5 & 3.2 & $-14.4(0.6)$ & -3.4 & 1.5 & -12.3 \\
\hline 9 & 0.8 & 1.6 & $-14.6(0.0)$ & -3.1 & 2.5 & -14.2 \\
\hline 10 & 0.3 & 21 & $-14.6(0.0)$ & -0.2 & 1 & -14.9 \\
\hline \multicolumn{7}{|c|}{ Conformation -7} \\
\hline 1 & 39.8 & 26.6 & $-16.0(1.3)$ & -2.4 & 1.6 & -16.6 \\
\hline 2 & 27.7 & 15.8 & $-15.8(1.0)$ & -3.4 & 1.3 & -13.4 \\
\hline 3 & 9.3 & 16.2 & $-15.1(1.0)$ & -2.2 & 1.4 & -14.3 \\
\hline 4 & 7.5 & 11.9 & $-15.5(0.4)$ & -2.3 & 1.5 & -14.2 \\
\hline 5 & 6.6 & 21.1 & $-15.1(0.8)$ & -2.1 & 1.2 & -14.2 \\
\hline 6 & 5.3 & 17.7 & $-15.0(0.3)$ & -2.3 & 1.7 & -14.2 \\
\hline 7 & 2.9 & 17.8 & $-15.0(0.4)$ & -0.3 & 0.5 & -14.8 \\
\hline 8 & 0.4 & 4.1 & $-14.8(0.6)$ & -4.2 & 4.7 & -15.3 \\
\hline
\end{tabular}




\begin{tabular}{ccccccc}
9 & 0.4 & 0.5 & $-14.7(0.3)$ & 0 & 1.2 & -15.8 \\
10 & 0.2 & 0 & $-14.6(0.4)$ & -2.2 & 2.7 & -15.1 \\
\hline
\end{tabular}


Table S6: Results of the BD rigid body docking of three different conformations of collagenase to collagen. Docking was performed for seven different conformations of the truncated collagen model generated by MD simulation. The encounter complexes generated in the BD simulations were clustered and ranked according to size. The values computed for the top ten clusters are given with the column header $(1,2,3)$ denoting the collagenase conformation. The RMSD was calculated between the structures of collagenase present in each cluster. The interaction energy is the sum of electrostatic interaction (ElE), electrostatic desolvation (ElDesE), and nonpolar (np) components. The values for the representative structure in each cluster, as well as the average value of the total interaction energy in the cluster (ClAE) (with standard deviation in brackets), are given. All energies are in $\mathrm{kcal} / \mathrm{mol}$.

\begin{tabular}{|c|c|c|c|c|c|c|c|c|c|c|c|c|c|c|c|c|c|c|}
\hline \multirow[t]{2}{*}{$\begin{array}{c}\text { Cluster } \\
\text { No. }\end{array}$} & \multicolumn{3}{|c|}{ Cluster Size (\%) } & \multicolumn{3}{|c|}{ RMSD $(\AA)$} & \multicolumn{3}{|c|}{$\begin{array}{c}\text { ClAE } \\
(\mathrm{kcal} / \mathrm{mol})\end{array}$} & \multicolumn{3}{|c|}{$\begin{array}{c}\text { ElE } \\
\text { (kcal/mol) }\end{array}$} & \multicolumn{3}{|c|}{$\begin{array}{c}\text { ElDesE } \\
(\mathrm{kcal} / \mathrm{mol})\end{array}$} & \multicolumn{3}{|c|}{$\begin{array}{c}\mathrm{np} \\
\text { (kcal/mol) }\end{array}$} \\
\hline & 1 & 2 & 3 & 1 & 2 & 3 & 1 & 2 & 3 & 1 & 2 & 3 & 1 & 2 & 3 & 1 & 2 & 3 \\
\hline \multicolumn{19}{|c|}{ Conformation 1} \\
\hline 1 & 33.03 & 38.08 & 43.85 & 11.95 & 28.19 & 20.53 & $-25.8(1.6)$ & $-20.5(1.0)$ & $-21.4(0.6)$ & -3.03 & -4.37 & -4.36 & 1.35 & 1.52 & 4.22 & -20.97 & -16.86 & -20.27 \\
\hline 2 & 21.67 & 30.43 & 16.83 & 9.12 & 15.40 & 7.29 & $-23.9(0.7)$ & $-20.4(0.8)$ & $-21.5(0.5)$ & -3.92 & -4.13 & -4.53 & 3.59 & 1.97 & 3.60 & -22.56 & -17.05 & -20.00 \\
\hline 3 & 15.36 & 13.44 & 11.02 & 22.32 & 19.16 & 10.28 & $-24.3(1.3)$ & $-20.2(0.5)$ & $-21.9(1.0)$ & -3.65 & -1.51 & -7.72 & 1.95 & 1.97 & 4.74 & -21.44 & -19.62 & -20.36 \\
\hline 4 & 12.74 & 6.67 & 10.19 & 19.11 & 1.18 & 13.14 & $-23.6(0.8)$ & $-20.5(0.7)$ & $-21.0(0.5)$ & -4.76 & -2.55 & -5.94 & 2.58 & 3.71 & 3.11 & -20.93 & -22.46 & -17.60 \\
\hline 5 & 9.00 & 4.26 & 6.51 & 5.08 & 27.12 & 3.98 & $-24.1(0.7)$ & $-19.8(0.5)$ & $-21.2(0.4)$ & -3.04 & -4.32 & -4.76 & 2.29 & 2.78 & 2.91 & -22.87 & -17.60 & -18.70 \\
\hline 6 & 5.86 & 2.76 & 4.46 & 15.30 & 13.35 & 10.58 & $-23.2(0.4)$ & $-19.7(0.4)$ & $-21.0(0.5)$ & -3.87 & -0.11 & -3.84 & 2.30 & 2.72 & 2.71 & -21.04 & -22.53 & -19.32 \\
\hline 7 & 0.68 & 2.33 & 3.20 & 8.00 & 15.57 & 2.00 & $-23.0(0.2)$ & $-20.2(0.7)$ & $-21.2(0.4)$ & -1.54 & -3.16 & -6.32 & 2.62 & 3.78 & 3.33 & -23.83 & -21.97 & -17.58 \\
\hline 8 & 0.64 & 1.63 & 3.01 & 6.47 & 11.26 & 3.80 & $-23.3(0.4)$ & $-20.0(0.5)$ & $-20.8(0.4)$ & -4.96 & 0.77 & -6.70 & 3.70 & 1.17 & 7.59 & -21.79 & -21.49 & -21.60 \\
\hline 9 & 0.53 & 0.27 & 0.88 & 0.70 & 0.19 & 4.72 & $-22.7(0.1)$ & $-19.3(0.2)$ & $-20.6(0.1)$ & -0.62 & -0.07 & -4.70 & 2.08 & 0.84 & 2.71 & -24.41 & -20.29 & -18.43 \\
\hline 10 & 0.49 & 0.14 & 0.05 & 13.62 & 0.28 & 0.00 & $-22.7(0.1)$ & $-19.6(0.3)$ & $-20.9(0.0)$ & -4.86 & -0.76 & -6.00 & 4.11 & 1.84 & 4.08 & -21.88 & -20.96 & -19.01 \\
\hline \multicolumn{19}{|c|}{ Conformation 2} \\
\hline 1 & 27.85 & 39.54 & 33.01 & 22.73 & 23.77 & 20.63 & $-25.1(1.8)$ & $-20.3(0.9)$ & $-20.8(0.6)$ & -6.02 & -1.66 & -5.53 & 2.00 & 1.80 & 3.00 & -20.20 & -21.52 & -18.91 \\
\hline 2 & 25.19 & 22.38 & 25.11 & 1.64 & 28.79 & 19.01 & $-25.3(0.9)$ & $-20.2(0.9)$ & $-21.7(1.1)$ & -5.91 & -1.05 & -3.51 & 4.36 & 1.09 & 1.89 & -24.83 & -19.29 & -18.66 \\
\hline 3 & 14.37 & 14.83 & 22.31 & 3.34 & 6.53 & 19.18 & $-24.8(0.8)$ & $-20.1(0.6)$ & $-20.8(0.6)$ & -3.33 & -3.04 & -6.91 & 1.63 & 3.03 & 3.35 & -24.15 & -18.95 & -16.37 \\
\hline
\end{tabular}




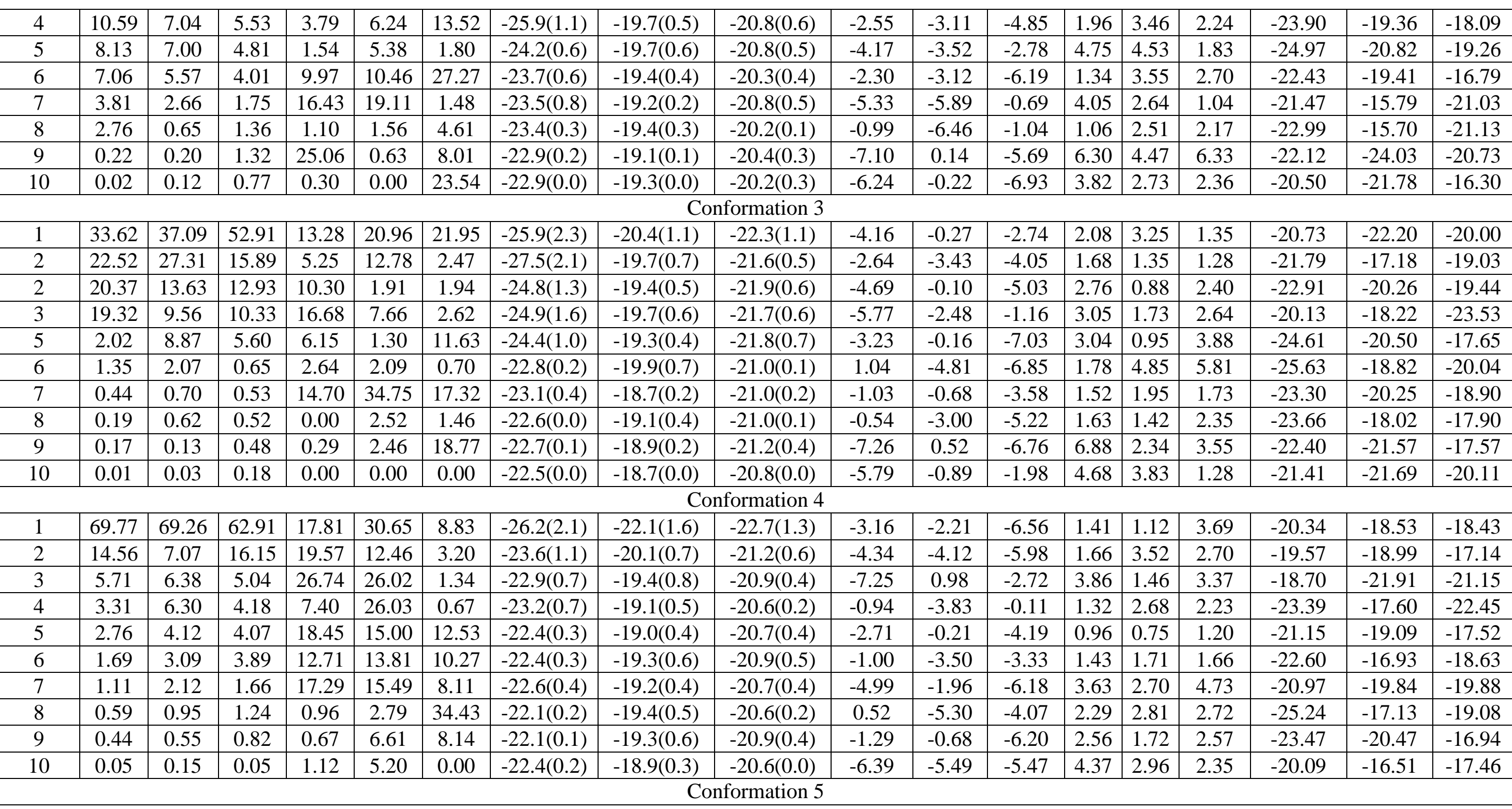




\begin{tabular}{|c|c|c|c|c|c|c|c|c|c|c|c|c|c|c|c|c|c|c|}
\hline 1 & 59.40 & 53.71 & 52.55 & 13.22 & 12.10 & 21.43 & $-25.8(2.1)$ & $-20.2(1.0)$ & $-21.9(1.5)$ & -5.42 & -4.32 & -7.43 & 2.59 & 1.90 & 4.05 & -19.22 & -18.05 & -16.59 \\
\hline 2 & 19.29 & 17.07 & 23.18 & 6.72 & 18.56 & 21.29 & $-24.0(1.3)$ & $-19.3(0.5)$ & $-20.5(0.6)$ & -2.54 & -3.35 & -6.74 & 1.27 & 1.26 & 4.06 & -21.63 & -16.66 & -17.02 \\
\hline 3 & 17.01 & 12.29 & 9.62 & 9.75 & 20.53 & 1.07 & $-23.8(0.9)$ & $-20.2(1.2)$ & $-20.3(0.4)$ & -6.62 & 0.00 & -0.45 & 3.61 & 2.09 & 2.63 & -19.61 & -20.94 & -23.16 \\
\hline 4 & 1.86 & 5.15 & 6.06 & 15.10 & 9.25 & 18.85 & $-23.1(0.7)$ & $-20.1(1.0)$ & $-19.9(0.2)$ & -2.43 & -1.38 & -3.66 & 2.13 & 2.99 & 1.77 & -22.75 & -20.62 & -18.03 \\
\hline 5 & 1.27 & 4.74 & 4.80 & 18.89 & 26.33 & 1.88 & $-22.5(0.2)$ & $-19.2(0.5)$ & $-20.6(0.5)$ & -5.31 & -2.19 & -3.62 & 4.44 & 3.23 & 2.58 & -21.52 & -20.77 & -19.98 \\
\hline 6 & 0.47 & 2.62 & 2.18 & 3.28 & 8.94 & 23.02 & $-22.5(0.4)$ & $-18.8(0.3)$ & $-20.5(0.7)$ & -3.84 & -0.99 & -6.22 & 2.99 & 1.59 & 4.47 & -21.20 & -19.24 & -17.91 \\
\hline 7 & 0.35 & 2.12 & 0.82 & 6.45 & 20.96 & 12.94 & $-22.2(0.1)$ & $-18.8(0.3)$ & $-20.1(0.3)$ & -4.78 & -4.38 & -3.72 & 6.30 & 2.07 & 3.67 & -23.55 & -16.41 & -20.58 \\
\hline 8 & 0.19 & 1.50 & 0.43 & 22.88 & 1.15 & 16.57 & $-22.4(0.1)$ & $-19.1(0.2)$ & $-19.8(0.1)$ & -2.25 & -2.74 & -0.27 & 2.41 & 3.36 & 1.95 & -22.17 & -19.56 & -21.36 \\
\hline 9 & 0.12 & 0.73 & 0.23 & 0.47 & 12.92 & 0.00 & $-22.6(0.4)$ & $-18.7(0.1)$ & $-19.8(0.0)$ & -5.23 & -0.54 & -1.52 & 3.74 & 1.47 & 3.95 & -21.30 & -19.43 & -22.26 \\
\hline 10 & 0.06 & 0.08 & 0.13 & 0.00 & 0.00 & 0.60 & $-22.1(0.0)$ & $-18.5(0.0)$ & $-20.0(0.0)$ & -3.97 & -0.18 & -5.86 & 2.08 & 1.08 & 3.29 & -20.21 & -19.45 & -17.41 \\
\hline \multicolumn{19}{|c|}{ Conformation 6} \\
\hline 1 & 67.04 & 43.33 & 43.25 & 17.54 & 26.09 & 6.65 & $-24.9(1.7)$ & $-19.5(0.5)$ & $-21.8(1.1)$ & -2.95 & -4.37 & -7.09 & 1.21 & 1.52 & 3.52 & -20.20 & -16.27 & -17.07 \\
\hline 2 & 17.99 & 25.30 & 19.07 & 14.66 & 27.86 & 12.14 & $-25.2(1.7)$ & $-19.7(0.6)$ & $-20.6(0.5)$ & -2.89 & -2.52 & -6.43 & 1.72 & 2.13 & 2.49 & -21.88 & -18.38 & -16.08 \\
\hline 3 & 9.26 & 10.86 & 18.17 & 24.83 & 22.78 & 17.00 & $-23.6(1.0)$ & $-20.0(0.9)$ & $-21.2(1.0)$ & -4.17 & -2.44 & -5.71 & 3.57 & 2.54 & 2.62 & -22.67 & -19.15 & -17.14 \\
\hline 4 & 2.72 & 5.69 & 7.00 & 10.38 & 6.03 & 5.45 & $-23.3(1.0)$ & $-19.0(0.2)$ & $-20.6(0.4)$ & -5.57 & -0.41 & -4.94 & 6.49 & 2.19 & 1.94 & -23.59 & -21.35 & -17.81 \\
\hline 5 & 0.80 & 5.31 & 6.08 & 8.26 & 5.44 & 17.45 & $-22.7(0.5)$ & $-19.3(0.4)$ & $-21.7(1.0)$ & -3.06 & -2.15 & -6.87 & 2.33 & 1.59 & 3.02 & -22.11 & -18.65 & -16.13 \\
\hline 6 & 0.77 & 5.17 & 5.14 & 0.94 & 15.67 & 1.15 & $-22.6(0.3)$ & $-19.2(0.4)$ & $-23.9(1.3)$ & -3.14 & -5.60 & -4.01 & 2.93 & 2.38 & 3.60 & -22.81 & -15.65 & -24.80 \\
\hline 7 & 0.61 & 2.38 & 0.49 & 11.68 & 15.67 & 1.20 & $-22.5(0.4)$ & $-19.2(0.4)$ & $-20.7(0.4)$ & -2.86 & -2.93 & -5.90 & 2.93 & 2.80 & 4.24 & -23.08 & -19.21 & -19.29 \\
\hline 8 & 0.59 & 1.17 & 0.40 & 7.22 & 17.36 & 3.44 & $-22.0(0.2)$ & $-19.9(1.2)$ & $-20.7(0.5)$ & -5.92 & -2.70 & -4.47 & 3.93 & 4.25 & 5.75 & -20.12 & -21.46 & -22.13 \\
\hline 9 & 0.19 & 0.75 & 0.37 & 0.30 & 1.33 & 0.15 & $-22.5(0.2)$ & $-18.8(0.2)$ & $-19.9(0.0)$ & -0.45 & -3.06 & -3.68 & 1.99 & 4.50 & 1.82 & -24.12 & -20.10 & -18.07 \\
\hline 10 & 0.02 & 0.03 & 0.03 & 0.00 & 0.00 & 0.00 & $-22.2(0.0)$ & $-19.2(0.0)$ & $-20.0(0.0)$ & -6.62 & -3.70 & -2.65 & 5.11 & 4.10 & 4.24 & -20.68 & -19.57 & -21.61 \\
\hline \multicolumn{19}{|c|}{ Conformation 7} \\
\hline 1 & 32.78 & 41.78 & 32.29 & 15.68 & 16.21 & 15.53 & $-24.5(1.9)$ & $-19.5(0.9)$ & $-21.5(0.8)$ & -4.42 & -4.15 & -7.08 & 2.38 & 2.06 & 4.19 & -25.60 & -17.84 & -17.15 \\
\hline 2 & 25.76 & 35.87 & 30.56 & 5.05 & 36.58 & 11.38 & $-24.2(0.9)$ & $-19.2(1.1)$ & $-20.8(0.6)$ & -2.60 & -3.52 & -5.13 & 1.79 & 2.42 & 2.37 & -24.54 & -16.96 & -17.42 \\
\hline 3 & 20.88 & 6.95 & 14.07 & 2.03 & 19.68 & 9.25 & $-24.9(1.3)$ & $-19.0(0.5)$ & $-20.9(0.6)$ & -4.44 & -0.11 & -4.76 & 2.52 & 1.35 & 2.14 & -22.17 & -19.52 & -17.52 \\
\hline 4 & 9.62 & 5.66 & 12.79 & 23.02 & 6.09 & 24.37 & $-22.8(0.4)$ & $-18.5(0.4)$ & $-20.7(0.4)$ & -6.94 & -4.10 & -6.71 & 4.41 & 1.74 & 3.37 & -19.58 & -15.80 & -17.05 \\
\hline 6 & 2.93 & 4.60 & 6.74 & 1.31 & 15.61 & 12.83 & $-23.8(0.5)$ & $-18.3(0.3)$ & $-20.6(0.4)$ & -4.57 & -5.94 & -4.26 & 2.68 & 2.61 & 2.78 & -21.85 & -15.08 & -18.87 \\
\hline 7 & 2.64 & 3.78 & 3.04 & 7.99 & 34.69 & 9.55 & $-22.6(0.4)$ & $-18.8(0.5)$ & $-22.2(1.4)$ & -4.08 & -3.28 & -1.75 & 5.27 & 3.85 & 2.93 & -24.33 & -19.76 & -22.04 \\
\hline 5 & 2.55 & 0.81 & 0.41 & 18.55 & 5.04 & 1.84 & $-22.6(0.4)$ & $-18.3(0.3)$ & $-20.1(0.1)$ & -3.66 & -0.12 & -4.28 & 3.07 & 1.77 & 1.96 & -21.54 & -20.28 & -17.69 \\
\hline 8 & 2.46 & 0.37 & 0.06 & 1.42 & 14.64 & 0.00 & $-23.7(1.0)$ & $-18.2(0.3)$ & $-20.0(0.0)$ & -2.04 & -0.18 & -5.22 & 2.95 & 1.34 & 3.44 & -25.08 & -19.45 & -18.25 \\
\hline
\end{tabular}




\begin{tabular}{c|c|c|c|c|c|c|c|c|c|c|c|c|c|c|c|c|c|c|c|c|}
\hline 9 & 0.24 & 0.15 & 0.05 & 1.21 & 0.00 & 0.00 & $-22.3(0.2)$ & $-18.2(0.0)$ & $-20.0(0.0)$ & -0.66 & 0.05 & -3.04 & 1.61 & 1.94 & 2.52 & -23.44 & -20.23 & -19.51 \\
\hline 10 & 0.14 & 0.02 & 0.00 & 15.19 & 0.00 & 0.75 & $-22.3(0.2)$ & $-18.1(0.0)$ & $-20.2(0.1)$ & -1.59 & -4.35 & -3.99 & 4.77 & 3.23 & 2.63 & -25.76 & -16.97 & -18.94 \\
\hline
\end{tabular}


Table S7: Results of the BD rigid body docking of MMP-1 to R55A (P8') mutant collagen. Docking was performed for seven different conformations of the truncated collagen model generated by MD simulation. The encounter complexes generated in the BD simulations were clustered and ranked according to size. The RMSD was calculated between the structures of HPX present in each cluster. The interaction energy is the sum of electrostatic interaction (ElE), electrostatic desolvation (ElDesE) and nonpolar (np) components. The value for the representative structure in each cluster, as well as the average values of the total interaction energy (ClAE) (with standard deviation in brackets) and its components, are given. All energies are in $\mathrm{kcal} / \mathrm{mol}$.

\begin{tabular}{|c|c|c|c|c|c|c|}
\hline Cluster No. & $\begin{array}{c}\text { Cluster Size } \\
\text { (\%) }\end{array}$ & RMSD ( $\AA)$ & ClAE & ElE & ElDesE & np \\
\hline \multicolumn{7}{|c|}{ Conformation 1} \\
\hline 1 & 43.8 & 16.4 & $-19.9(1.3)$ & -5.2 & 2.5 & -16.2 \\
\hline 2 & 24.4 & 6.3 & $-20.8(1.6)$ & -5.9 & 2.6 & -17.1 \\
\hline 3 & 17.3 & 22.9 & $-18.6(0.5)$ & -3.1 & 1.7 & -16.8 \\
\hline 4 & 10.5 & 19.8 & $-19.0(0.6)$ & -2.2 & 2.8 & -19.6 \\
\hline 5 & 2.9 & 1.4 & $-19.2(0.6)$ & -4.6 & 2.9 & -17.4 \\
\hline 6 & 0.4 & 2.4 & $-18.4(0.3)$ & -0.5 & 0.9 & -18.6 \\
\hline 7 & 0.3 & 25.5 & $-18.4(0.6)$ & -1.3 & 1.2 & -18.2 \\
\hline 8 & 0.3 & 0.3 & $-18.2(0.1)$ & -3.1 & 3.1 & -18.3 \\
\hline 9 & 0 & 0 & $-18.1(0.0)$ & -3.3 & 4.2 & -19.1 \\
\hline 10 & 0 & 0 & $-18.4(0.0)$ & -3 & 4 & -19.4 \\
\hline \multicolumn{7}{|c|}{ Conformation 2} \\
\hline 1 & 21.8 & 20.9 & $-19.4(0.6)$ & -3.6 & 2.5 & -17.6 \\
\hline 2 & 21 & 15.8 & $-19.5(0.6)$ & -3.5 & 2.4 & -17.9 \\
\hline 3 & 20.8 & 12.9 & $-20.2(1.3)$ & -4.1 & 2.3 & -16.8 \\
\hline 4 & 18.3 & 5.1 & $-20.3(1.2)$ & -3.2 & 2.3 & -18.2 \\
\hline 5 & 11.3 & 5.8 & $-20.4(0.9)$ & -3.6 & 3.4 & -18.6 \\
\hline 6 & 3.6 & 8.3 & $-19.0(0.4)$ & -5.4 & 2.9 & -16.5 \\
\hline 7 & 1.5 & 1.2 & $-18.8(0.3)$ & -5.3 & 3.3 & -16.7 \\
\hline 8 & 1.4 & 15.2 & $-18.9(0.5)$ & -3.4 & 3.4 & -18.5 \\
\hline 9 & 0.3 & 17.2 & $-18.8(0.4)$ & -4.5 & 2.5 & -16.9 \\
\hline 10 & 0.1 & 0 & $-18.5(0.0)$ & -2.8 & 4.4 & -20.1 \\
\hline \multicolumn{7}{|c|}{ Conformation 3} \\
\hline 1 & 39.1 & 26.1 & $-19.1(1.2)$ & -4.8 & 2.7 & -16.1 \\
\hline 2 & 20.2 & 35.7 & $-18.8(1.3)$ & -3.2 & 1.6 & -17.6 \\
\hline 3 & 11.1 & 25 & $-18.1(0.4)$ & -5 & 3.9 & -16.9 \\
\hline 4 & 9.5 & 5.6 & $-19.9(1.3)$ & -4.4 & 2.8 & -18 \\
\hline 5 & 7.3 & 8 & $-18.6(0.6)$ & -4.3 & 6.1 & -21.4 \\
\hline 6 & 5.6 & 21.7 & $-18.3(0.5)$ & -2.5 & 2.3 & -17.4 \\
\hline 7 & 5.6 & 3.2 & $-18.1(0.5)$ & -1.3 & 4.6 & -21.4 \\
\hline 8 & 1.4 & 17.2 & $-18.1(0.3)$ & -4.9 & 2.9 & -15.8 \\
\hline 9 & 0.1 & 0 & $-17.6(0.0)$ & -2.3 & 2.1 & -17.4 \\
\hline
\end{tabular}




\begin{tabular}{|c|c|c|c|c|c|c|}
\hline 10 & 0.1 & 0 & $-18.0(0.0)$ & -1 & 2.4 & -19.3 \\
\hline \multicolumn{7}{|c|}{ Conformation 4} \\
\hline 1 & 47.9 & 10.6 & $-19.7(1.0)$ & -4.2 & 2.8 & -17.1 \\
\hline 2 & 17.4 & 15.9 & $-19.4(1.0)$ & -3.5 & 2.2 & -17.1 \\
\hline 3 & 12.4 & 1.9 & $-20.3(1.1)$ & -3.6 & 3.2 & -21 \\
\hline 4 & 7 & 3.5 & $-18.8(0.5)$ & -3.3 & 2 & -17.4 \\
\hline 5 & 6.1 & 3.3 & $-19.0(0.5)$ & -3.6 & 2.5 & -17.6 \\
\hline 6 & 3.9 & 16.3 & $-18.7(0.4)$ & -5 & 2.2 & -15.7 \\
\hline 7 & 2.5 & 5 & $-19.0(0.4)$ & -2.9 & 2.7 & -19.1 \\
\hline 8 & 1.5 & 2.4 & $-18.6(0.3)$ & -2.2 & 2 & -18.5 \\
\hline 9 & 1.3 & 1.2 & $-19.3(0.6)$ & -3.3 & 3.2 & -19.9 \\
\hline 10 & 0.1 & 0 & $-18.3(0.0)$ & -4.6 & 2 & -15.7 \\
\hline \multicolumn{7}{|c|}{ Conformation 5} \\
\hline 1 & 37.2 & 14.2 & $-20.8(1.4)$ & -3.1 & 2.1 & -17.7 \\
\hline 2 & 34 & 18.3 & $-19.8(0.7)$ & -5.2 & 2.7 & -16.7 \\
\hline 3 & 10.9 & 8.5 & $-19.6(0.6)$ & -4.5 & 3.3 & -17.6 \\
\hline 4 & 5.6 & 4.3 & $-20.0(0.7)$ & -4.5 & 2.5 & -16.8 \\
\hline 5 & 4.8 & 19.3 & $-19.7(0.9)$ & -2.5 & 1.9 & -18.1 \\
\hline 6 & 4.6 & 10.4 & $-19.6(0.6)$ & -4.8 & 3.1 & -18.7 \\
\hline 7 & 1.3 & 6.3 & $-19.3(0.6)$ & -2.2 & 2.7 & -19.5 \\
\hline 8 & 1 & 5.5 & $-20.5(1.1)$ & -4.3 & 3.9 & -21.6 \\
\hline 9 & 0.5 & 0.8 & $-20.1(0.4)$ & -4.1 & 5.8 & -21.6 \\
\hline 10 & 0.2 & 4.5 & $-19.1(0.2)$ & -5.3 & 2.1 & -15.9 \\
\hline \multicolumn{7}{|c|}{ Conformation 6} \\
\hline 1 & 16.9 & 13 & $-19.1(1.1)$ & -3.1 & 1.8 & -17.4 \\
\hline 2 & 16.1 & 16.8 & $-18.1(0.5)$ & -4.5 & 3.9 & -17.1 \\
\hline 3 & 14.1 & 3.5 & $-18.9(1.1)$ & -2.9 & 2.2 & -17.8 \\
\hline 4 & 12.1 & 23 & $-18.8(1.2)$ & -4.8 & 2.1 & -15.8 \\
\hline 5 & 11.5 & 15.5 & $-19.0(1.3)$ & -1.3 & 1.5 & -18.2 \\
\hline 6 & 10.3 & 19.6 & $-18.2(0.7)$ & -4 & 1.5 & -15 \\
\hline 7 & 9.3 & 2.4 & $-18.7(0.8)$ & -5.8 & 4.4 & -18.8 \\
\hline 8 & 4.7 & 16.1 & $-18.2(0.5)$ & -3.2 & 2.4 & -17.2 \\
\hline 9 & 2.8 & 2.7 & $-18.3(0.4)$ & -3.2 & 4.8 & -19.7 \\
\hline 10 & 2.3 & 2.4 & $-18.7(0.5)$ & -4.5 & 2.3 & -17 \\
\hline \multicolumn{7}{|c|}{ Conformation 7} \\
\hline 1 & 48.3 & 17.6 & $-19.9(1.0)$ & -4.3 & 2.2 & -17.3 \\
\hline 2 & 19.5 & 17.1 & $-19.8(0.9)$ & -3.1 & 2.3 & -19.1 \\
\hline 3 & 11.1 & 2.2 & $-20.3(0.8)$ & -6.3 & 7.2 & -21.9 \\
\hline 4 & 8.5 & 15.3 & $-19.5(0.8)$ & -4.1 & 3.4 & -18.2 \\
\hline 5 & 6 & 8.5 & $-20.0(1.0)$ & -5.3 & 2.6 & -16 \\
\hline 6 & 2.8 & 2 & $-19.2(0.4)$ & -4.4 & 3 & -17.5 \\
\hline 7 & 2 & 26.3 & $-19.4(0.6)$ & -4 & 1.9 & -16.7 \\
\hline 8 & 0.9 & 6.4 & $-19.0(0.3)$ & -3.9 & 4.3 & -19 \\
\hline
\end{tabular}




\begin{tabular}{ccccccc}
9 & 0.6 & 0.9 & $-19.1(0.3)$ & -1.5 & 2.4 & -19.9 \\
10 & 0.4 & 1.2 & $-18.8(0.1)$ & -4.9 & 4.2 & -18.1 \\
\hline
\end{tabular}


Table S8: Results of the BD rigid body docking of MMP-1 to R67A (P20') mutant collagen. Docking was performed for seven different conformations of the truncated collagen model generated by MD simulation. The encounter complexes generated in the BD simulations were clustered and ranked according to size. The RMSD was calculated between the structures of HPX present in each cluster. The interaction energy is the sum of electrostatic interaction (ElE), electrostatic desolvation (ElDesE) and nonpolar (np) components. The value for the representative structure in each cluster, as well as the average values of the total interaction energy (ClAE) (with standard deviation in brackets) and its components, are given. All energies are in $\mathrm{kcal} / \mathrm{mol}$.

\begin{tabular}{|c|c|c|c|c|c|c|}
\hline $\begin{array}{l}\text { Cluster } \\
\text { No. }\end{array}$ & $\begin{array}{c}\text { Cluster Size } \\
(\%)\end{array}$ & $\operatorname{RMSD}(\AA)$ & ClAE & ElE & EIDesE & np \\
\hline \multicolumn{7}{|c|}{ Conformation 1} \\
\hline 1 & 24.3 & 17.3 & $-18.1(1.1)$ & -1.6 & 1.3 & -17.1 \\
\hline 2 & 23.1 & 27.6 & $-17.3(0.6)$ & -1.4 & 1 & -16.7 \\
\hline 3 & 18.2 & 3.8 & $-19.1(1.1)$ & -2.1 & 4.9 & -23.3 \\
\hline 4 & 17.9 & 25.5 & $-18.5(1.4)$ & -1.2 & 2.5 & -17.9 \\
\hline 5 & 9.6 & 1.6 & $-17.3(0.4)$ & -4.3 & 2.1 & -15.7 \\
\hline 6 & 2.6 & 2.3 & $-16.9(0.4)$ & -0.2 & 3 & -19.6 \\
\hline 7 & 2.2 & 10.2 & $-16.8(0.3)$ & -5.5 & 2.6 & -13.7 \\
\hline 8 & 1.4 & 8.7 & $-18.1(0.9)$ & -0.1 & 1.4 & -20.4 \\
\hline 9 & 0.7 & 10.4 & $-16.8(0.4)$ & -2.2 & 1.2 & -15.5 \\
\hline 10 & 0 & 0 & $-16.6(0.0)$ & -2 & 3.1 & -17.6 \\
\hline \multicolumn{7}{|c|}{ Conformation 2} \\
\hline 1 & 38.9 & 37.5 & $-17.8(0.6)$ & -3.2 & 1.8 & -15.9 \\
\hline 2 & 27.7 & 35.8 & $-18.4(1.2)$ & -4.2 & 3.9 & -17.3 \\
\hline 3 & 7.5 & 6 & $-17.9(0.6)$ & -5 & 3.4 & -16.3 \\
\hline 4 & 7.4 & 15.4 & $-18.1(0.9)$ & -3.7 & 2 & -17 \\
\hline 5 & 6 & 14.1 & $-17.8(0.6)$ & -4.9 & 2.6 & -15.5 \\
\hline 6 & 3.9 & 2.2 & $-17.3(0.3)$ & -1.8 & 2.9 & -18.6 \\
\hline 7 & 3.7 & 25.9 & $-17.5(0.3)$ & -1.3 & 2.9 & -18.5 \\
\hline 8 & 2.5 & 2.6 & $-17.3(0.2)$ & 0.5 & 1.7 & -19.4 \\
\hline 9 & 2.4 & 23.3 & $-18.0(0.8)$ & -0.8 & 2.8 & -20 \\
\hline 10 & 0.1 & 0 & $-17.0(0.0)$ & -0.6 & 3.2 & -19.6 \\
\hline \multicolumn{7}{|c|}{ Conformation 3} \\
\hline 1 & 31.1 & 31.4 & $-18.0(1.2)$ & -0.3 & 1.6 & -18.1 \\
\hline 2 & 24.4 & 27.7 & $-17.6(0.8)$ & -3.9 & 2.4 & -15.5 \\
\hline 3 & 10.6 & 19.3 & $-17.4(0.4)$ & -4.8 & 3.3 & -15.4 \\
\hline 4 & 10.3 & 13.3 & $-17.9(0.6)$ & -2.2 & 2.9 & -18.6 \\
\hline 5 & 9.6 & 13.6 & $-18.1(0.9)$ & -3.2 & 2.5 & -16.3 \\
\hline 6 & 8.2 & 16.3 & $-17.5(0.6)$ & -3.2 & 1.9 & -16.2 \\
\hline 7 & 2.1 & 3.5 & $-17.9(0.6)$ & -1.4 & 1.6 & -17.7 \\
\hline 8 & 1.8 & 1.5 & $-17.8(0.5)$ & -0.1 & 1 & -19.3 \\
\hline 9 & 1.1 & 6 & $-17.6(0.5)$ & -2.2 & 2.1 & -18.3 \\
\hline
\end{tabular}




\begin{tabular}{|c|c|c|c|c|c|c|}
\hline 10 & 0.6 & 20 & $-16.9(0.1)$ & -1.1 & 2.8 & -18.6 \\
\hline \multicolumn{7}{|c|}{ Conformation 4} \\
\hline 1 & 33.1 & 4.9 & $-18.9(1.1)$ & -0.4 & 1.1 & -19.8 \\
\hline 2 & 31.2 & 39.5 & $-18.5(1.6)$ & -1.6 & 1.4 & -16.8 \\
\hline 3 & 13.4 & 13.3 & $-18.1(0.9)$ & -2.1 & 2.1 & -17.3 \\
\hline 4 & 13.4 & 10.2 & $-18.3(1.0)$ & -1.7 & 3.5 & -19.4 \\
\hline 5 & 3.4 & 16 & $-17.1(0.4)$ & -0.4 & 2.2 & -19.4 \\
\hline 6 & 2.2 & 4.7 & $-17.4(0.5)$ & -3.1 & 2.6 & -16.6 \\
\hline 7 & 1.8 & 9.5 & $-16.9(0.2)$ & -5.3 & 5.3 & -16.9 \\
\hline 8 & 1.1 & 17.8 & $-17.4(0.6)$ & -1.4 & 1.4 & -16.7 \\
\hline 9 & 0.4 & 5.6 & $-18.1(0.9)$ & -3.4 & 3 & -17.5 \\
\hline 10 & 0 & 0 & $-17.1(0.0)$ & -2.2 & 2.9 & -17.8 \\
\hline \multicolumn{7}{|c|}{ Conformation 5} \\
\hline 1 & 59.6 & 36.1 & $-18.5(0.9)$ & -5.1 & 2 & -14.3 \\
\hline 2 & 26.3 & 21.3 & $-18.5(0.8)$ & -0.1 & 2 & -19.5 \\
\hline 3 & 4.3 & 20.6 & $-18.2(0.7)$ & 0.1 & 0.7 & -18.4 \\
\hline 4 & 2.2 & 3 & $-18.2(0.5)$ & -4.4 & 3.4 & -16.9 \\
\hline 5 & 1.9 & 40.3 & $-17.8(0.3)$ & -3.7 & 2.2 & -16.7 \\
\hline 6 & 1.8 & 7.5 & $-18.7(0.6)$ & -5.2 & 3.9 & -17.9 \\
\hline 7 & 1.7 & 17.6 & $-18.1(0.6)$ & -4 & 3.7 & -17.3 \\
\hline 8 & 1.1 & 28.6 & $-18.0(0.5)$ & -0.9 & 1.3 & -18.7 \\
\hline 9 & 0.6 & 14.5 & $-17.5(0.3)$ & -3.7 & 5.6 & -20.4 \\
\hline 10 & 0.4 & 22.1 & $-17.7(0.2)$ & -2.7 & 2 & -17 \\
\hline \multicolumn{7}{|c|}{ Conformation 6} \\
\hline 1 & 41.7 & 30.7 & $-18.7(1.7)$ & -3.7 & 2.3 & -16.5 \\
\hline 2 & 38.5 & 13.5 & $-20.9(2.2)$ & -3.5 & 2.5 & -16 \\
\hline 3 & 8.7 & 19.8 & $-18.8(1.8)$ & -1.3 & 1.1 & -19 \\
\hline 4 & 7.7 & 22.6 & $-18.0(0.9)$ & -1.9 & 1.4 & -16.8 \\
\hline 5 & 1.5 & 6.1 & $-18.1(1.0)$ & -1.4 & 1.9 & -17.9 \\
\hline 6 & 0.6 & 1.7 & $-17.2(0.2)$ & 0.1 & 1.3 & -18.4 \\
\hline 7 & 0.5 & 0.6 & $-17.4(0.2)$ & -0.1 & 1.4 & -18.9 \\
\hline 8 & 0.4 & 17.5 & $-17.3(0.4)$ & -2.5 & 2.7 & -17.6 \\
\hline 9 & 0.4 & 31.8 & $-17.4(0.5)$ & -3.7 & 3.4 & -16.7 \\
\hline 10 & 0.1 & 0 & $-17.0(0.0)$ & -4.2 & 2 & -14.8 \\
\hline \multicolumn{7}{|c|}{ Conformation 7} \\
\hline 1 & 41.3 & 35.1 & $-19.6(1.6)$ & -4.3 & 2.4 & -15 \\
\hline 2 & 15.3 & 14.5 & $-18.0(0.8)$ & -4.3 & 5.4 & -18.4 \\
\hline 3 & 13.9 & 20.1 & $-18.1(0.8)$ & -2.2 & 3.4 & -18.4 \\
\hline 4 & 12.6 & 28.5 & $-17.4(0.5)$ & -5.7 & 2.7 & -14.2 \\
\hline 5 & 8.4 & 13.4 & $-18.4(1.0)$ & -0.3 & 2.5 & -20 \\
\hline 6 & 2.9 & 5.6 & $-17.5(0.6)$ & -2.4 & 3.2 & -17.9 \\
\hline 7 & 2.3 & 23.7 & $-17.3(0.4)$ & -3.7 & 3.5 & -17.1 \\
\hline 8 & 1.6 & 2.5 & $-17.4(0.5)$ & -0.5 & 1.1 & -17.6 \\
\hline
\end{tabular}




\begin{tabular}{ccccccr}
9 & 1 & 4.7 & $-17.5(0.5)$ & -4.2 & 3.2 & -16.3 \\
10 & 0.5 & 3.2 & $-17.4(0.6)$ & -3.2 & 2.3 & -16.5 \\
\hline
\end{tabular}




$\begin{array}{ll}\text { MMP-13 } & \text { Zebrafish } \\ \text { MMP-13 } & \text { coelacanth } \\ \text { MMP-13 } & \text { frog } \\ \text { MMP-13 } & \text { turtle } \\ \text { MMP-13 } & \text { Tasmanian devil } \\ \text { MMP-13 } & \text { Mouse } \\ \text { MMP-13 } & \text { Rat } \\ \text { MMP-13 } & \text { Bovine } \\ \text { MMP13 } & \text { RABIT } \\ \text { MMP13 } & \text { HUMAN } \\ \text { MMP-8 } & \text { frog } \\ \text { MMP1 } & \text { BovIN } \\ \text { MMP1 } & \text { Bullfrog } \\ \text { MMP1 } & \text { PIG } \\ \text { MMP1 } & \text { RABIT } \\ \text { MMP1 } & \text { HUMAN } \\ \text { MMP1 } & \text { HORSE } \\ \text { MMP-8 } & \text { Duckbill platypus } \\ \text { MMP-8 } & \text { Tasmanian devil } \\ \text { MMP-8 } & \text { Guinea pig } \\ \text { MMP-8 } & \text { RAT } \\ \text { MMP-8 } & \text { Mouse } \\ \text { MMP-8 } & \text { MOUSE } \\ \text { MMP-8 } & \text { BovIN } \\ \text { MMP-8 } & \text { SHEEP } \\ \text { MMP-8 } & \text { Little brown bat } \\ \text { MMP-8 } & \text { Small-eared galago } \\ \text { MMP-8 } & \text { marmoset } \\ \text { MMP-8 } & \text { Rhesus macaque } \\ \text { MMP-8 } & \text { Sumatran orangutan } \\ \text { MMP-8 } & \text { HUMAN } \\ \text { MMP-8 } & \text { gorilla } \\ \text { MMP-8 } & \text { RABIT } \\ \text { MMP-8 } & \text { Squirrel } \\ \text { Clustal } & \text { Consensus } \\ & \end{array}$

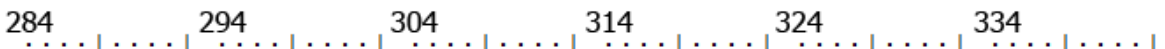
LDAVTMLRGE IMFFKNSFFW RSYPQSPDVE LQLIQSFWPE IPDNIDAAFE SVIQDKVFLI FDAITELRGE KMIFKDRFFW RQHPQMVDAE LTLIKSFWPE IPNRIDAAYE YSEKDRVFLF IDAITELRGE KMIFKDRFF' RVHPQMTDAE LVLIKSFWPE LPNKLDAAYE HPAKDLSYLF LDAITELRGE TMIFKDRFFW RLHPQMVEAE LVLIKSFWPE LPNKIDAAYE NPIKDHVIIF VDAITSLRGE TIIFKDRFFW RLHPOMIEPD LFLTKSFWPE LPNSIDAAYE NPAHDLIFIF LDAITSLRGE TMIFKDRFF' RLHPQQVEAE LFLTKSFWPE LPNHVDAAYE HPSRDLMFIF LDAITSLRGE TMIFKDRFFW RLHPQQVEPE LFLTKSFWPE LPNHVDAAYE HPSRDLMFIF LDAITSLRGE TLIFKDRFF' RLHPQQVEAE LFLTKSFGPE LPNRIDAAYE HPSHDLIFIF LDAITSLRGE TMIFKDRFF' RLHPQQVDAE LFLTKSFWPE LPNRIDAAYE HPARDLIFIF LDAITSLRGE TMIFKDRFFW RLHPQQVDAE LFLTKSFWPE LPNRIDAAYE HPSHDLIFIF FDAITTLRGE ILFFKHRSFW RKIPNKSEIE QHEIRTFWKT LPTGIQAAYE NOEKDQVFLF FDAITTIRGE VMFFKDRFYM RTNPLYPEVE LNFISVFWPQ LPNGLQAAYE VADRDEVRFF FDAITTVRGE LMFFKM---- RTNRFYPEVE L-_------ - - GLOAAYE MADRDEVRFF FDAITTLRGE LMFFKDRFYM RTNSFYPEVE LNFISVFWPQ VPNGLQAAYE IADRDEVRFF FDAITTIRGE IMFFKDRFYM RANPYYSEVE LNFISVFWPH LPNGLQAAYE VAHRDEILFF FDAITTIRGE VMFFKDRFYM RTNPFYPEVE LNFISVFWPQ LPNGLEAAYE FADRDEVRFF FDAITTIRGE VMFFKDRFYM RINPYYPEAE LNFISIFWPQ LPNGLQAAYE VSHRDEVRFF FDAITTLRGE IIFFKDKYFW RKHPOMTSVE FNSISLFWPT LPEGIOAAFE DKENDVVFLF FDAITTLRGE VIIFKDKYFW RKHRHIIRPE LHMISLFWPS LPSGIQAAFE DVANDLVFLF FDAVTTLRGE IFFFKDKFFW RRHPQQQVLD LNFISLFWPS LPNGIQAAYE DSDKDLVFIF FDAATTLRGE IYFFKDKYFW RRHPQLRTVD LNFISLFWPF LPNGLQAAYE DFDRDLVFLF FDATTTLRGE IYFFKDKYFW RRHPQLRTVD LNFISLFWPF LPNGLQAAYE DFDRDLVFLF FDATTTLRGE IYFFKDKYFW RRHPQLRTVD LNFISLFWPF LPNGLQAAYE DFDRDLVFLF FDAVTTLRGE ILFFKDKYVW RKHPRLRMAE FNFISLYWPS LPNGIHAAYE DFDRDLIFIF FDAVTTLRGE KLFFKDKYVW RRHPQLPMVE LNFISLYWPS LPNGIQAAYE DFDRDLIFIF FDAITTLRGE ILFFKDKYFW RRHPOLRRVE LNFISLFWPS LPTGIOAAYE DSDRDLVFLF FDAITTLRGE ILFFKDKYFW RKHPQLPTAE LNFISLFWPS LPNGIQAAYE DFERDLVFLF FDAITTLRGE IFFFKDKYFW RRHPQLQRVE MNFISLFWPS LPTGIQAAYE DFDRDLVFLF FDAITTLRGE ILFFKDEYFW RRHPHLQSVE MNFISLFWPS LPTGIOAAYE DFDRDLIFLF FDAITTLRGE ILFFKDKYFW RRHPQLQRIE MNFISLFWPS LPTGMQAAYE DFDRDLIFLF FDAITTLRGE ILFFKDRYFW RRHPQLORVE MNFISLFWPS LPTGIOAAYE DFDRDLIFLF FDAITTLRGE ILFFKDRYFW RRHPQLQRVE MNFISLFWPS LPTGIQAAYE DFDRDLIFLF FDAATTFRGE ILFFTDKYFW RRHPWLRRVE LNFISLFWPS LPNGIQAAYE DFDRDLVFLF FDAATTLRGE ILFFKDKYFW RRHPQLRLVE LNFISLFWPS LPNGIQAAYE DFDRDLVFLF

Figure S1: Part of a multiple sequence alignment of the HPX domain of classic collagenases. Residues that interact with collagen during rigid body docking are conserved charged residues shown in boxes. 

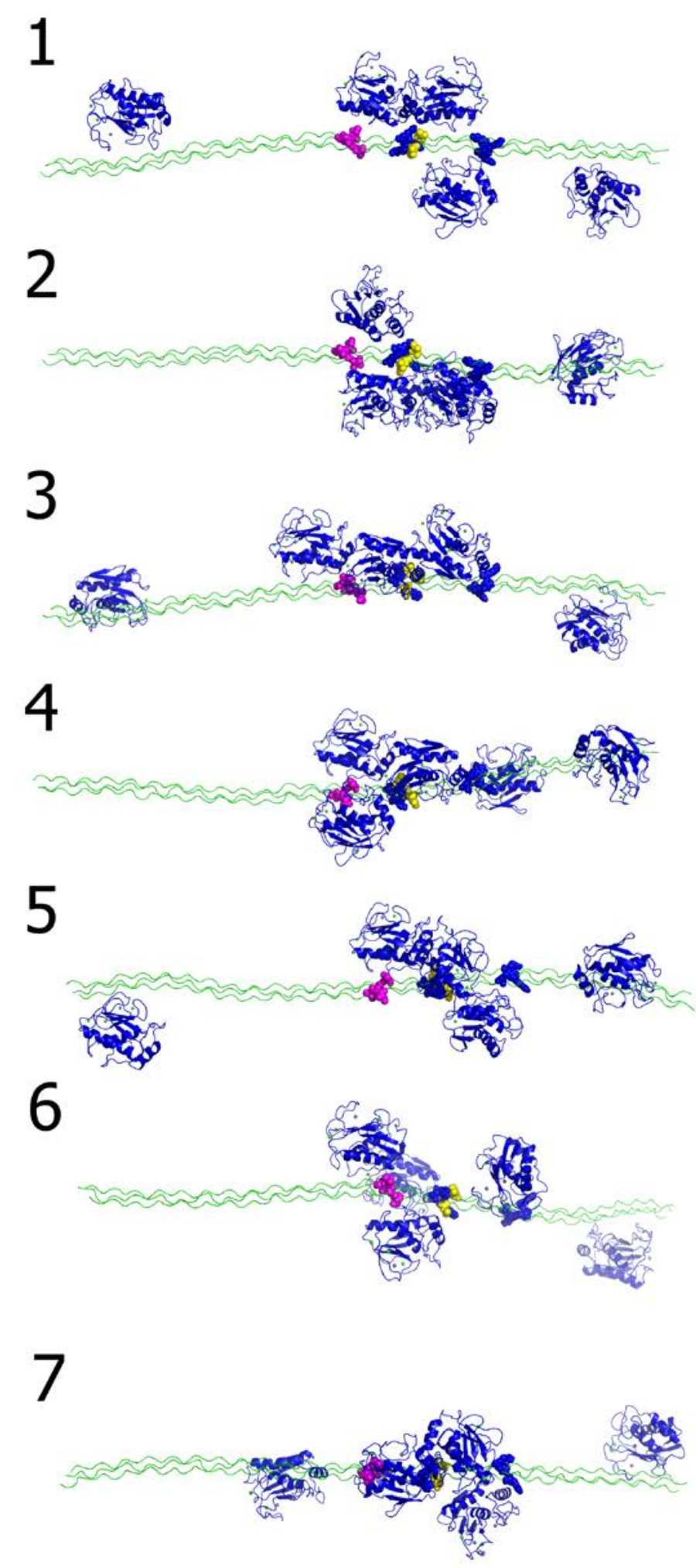

Figure S2. Schematic representation of the representatives of the five top-ranked encounter complex (EC) clusters identified by rigid body docking of the CAT domain (blue) of collagenase MMP-1 to 7 conformations of the truncated collagen model (green with the cleavage site in magenta and Arg P8' and P20' in blue). 


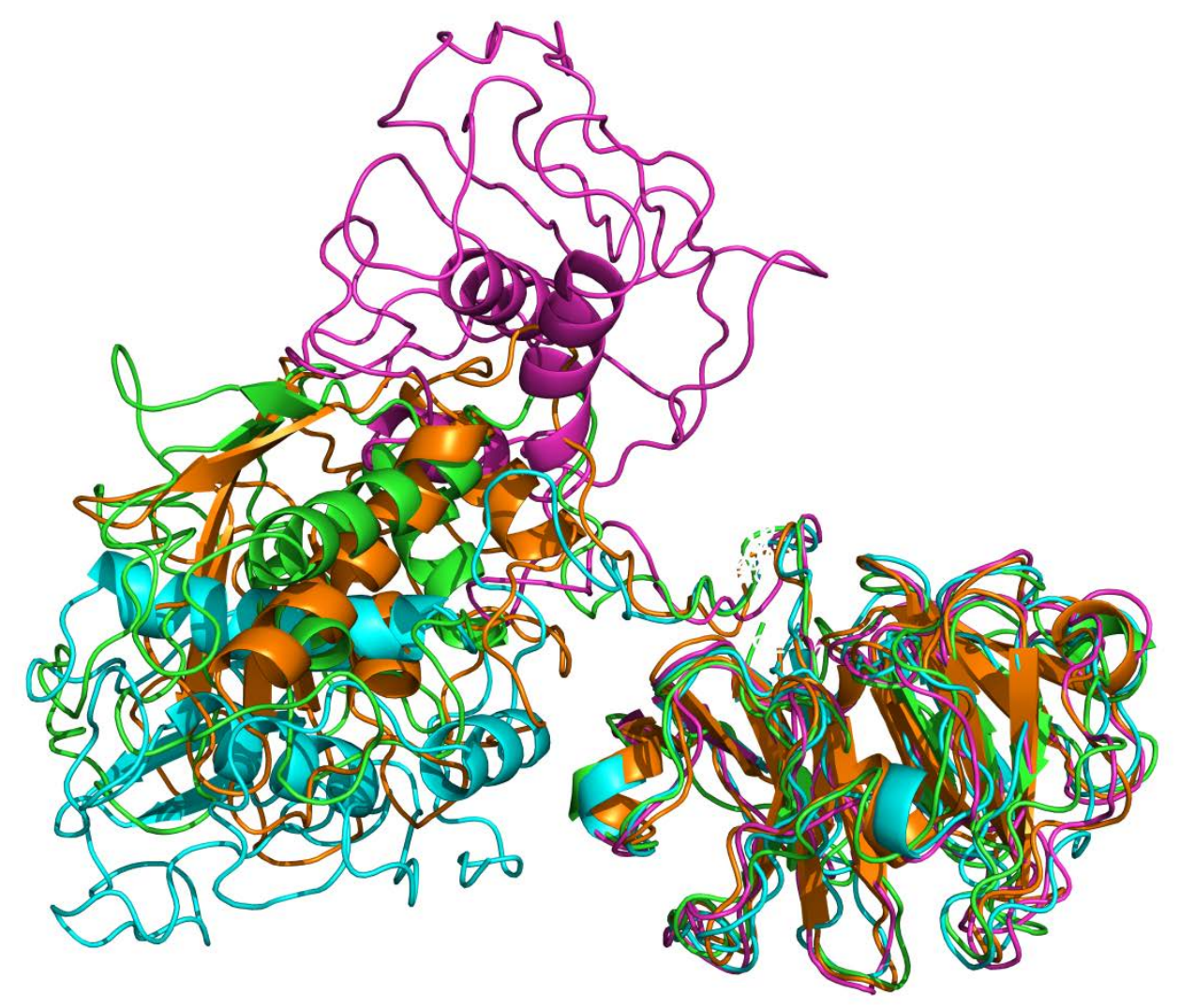

Figure S3. Three different conformations of MMP-1 generated using coarse-grained molecular dynamics simulations. The conformations are shown in cyan, green and pink and were aligned by superimposing the backbone atoms of the HPX domain of MMP-1. The crystal structure (PDB id 1FBL) is shown for reference in orange. 

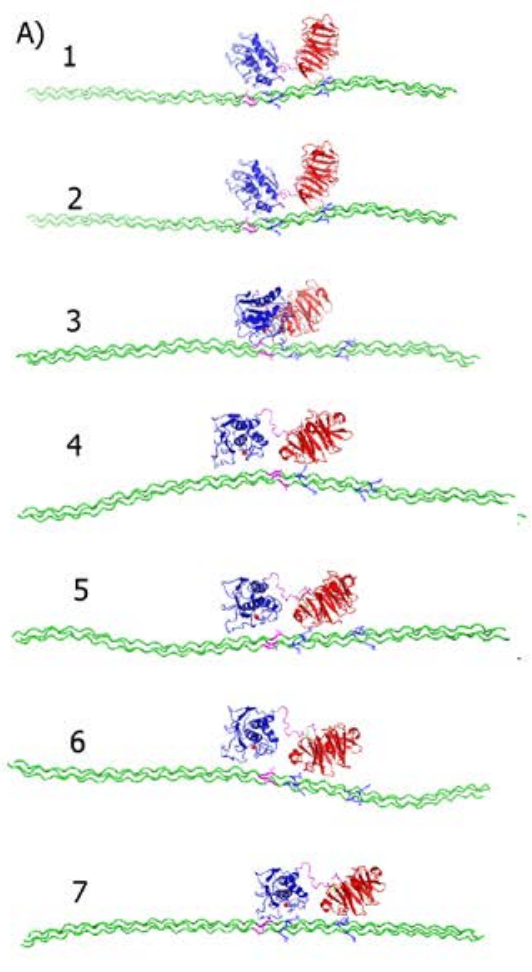

B)
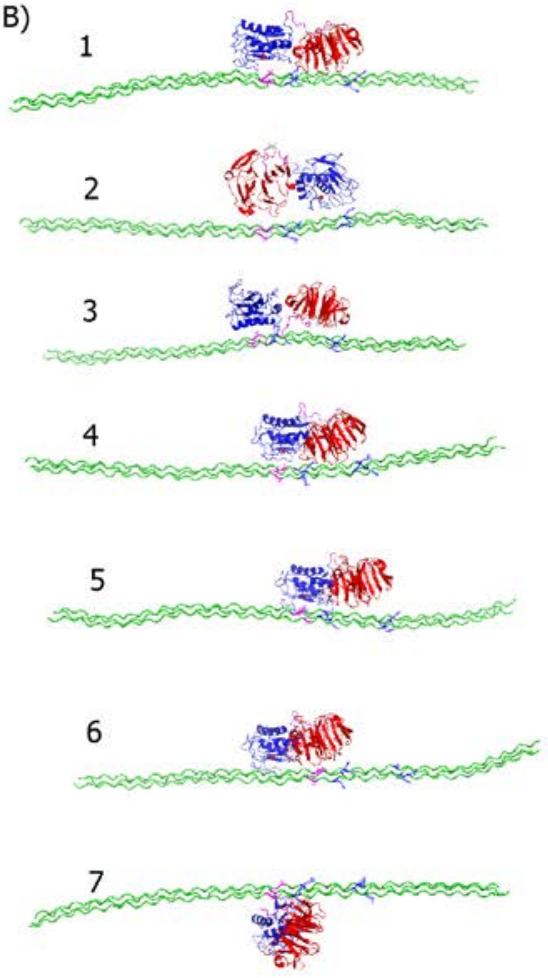

C) 1

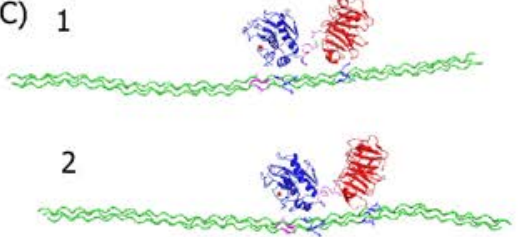

3
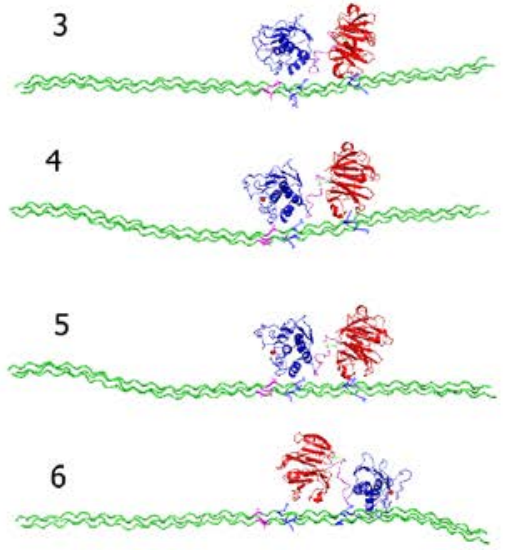

7

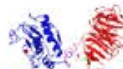

Figure S4. Schematic representation of the most populated encounter complexes (EC) identified by rigid body docking of the three different conformations (A:1, B:2 and C:3) of MMP-1 to 7 conformations (1 to 7) of the truncated collagen model. 


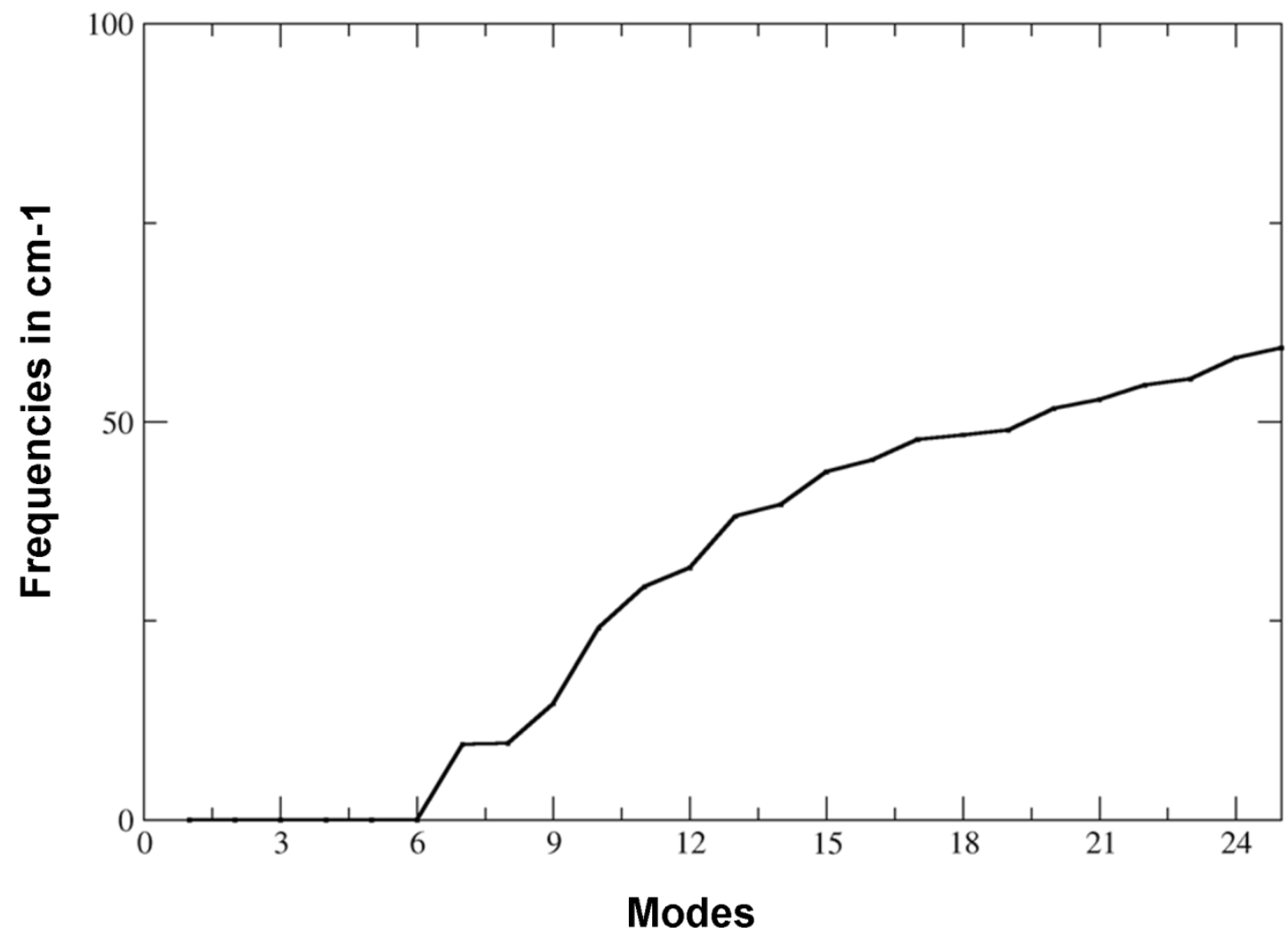

Figure S5. Normal mode analysis of MMP-1. Eigenvalues/frequencies of the 50 lowest frequency modes were calculated for an Elastic Network Model (ENM) using the NOMADRef webserver (http://lorentz.immstr.pasteur.fr/index1.php) (Lindahl et al., 2006). Here, the first 6 modes are translational and rotational modes. Modes 7 and 8 are referred to in the main article as modes 1 and 2, respectively, and correspond to the lowest frequency internal motion modes, see Figure 6 and Movies S1 and S2. 


C1A1 Homo sapiens
C1A1 Danio rerio
C1A1 Mus musculus
C1A1 Rattus norvegicus
C1A1 Bos taurus
C1A1 Oryzias latipes
C1A1 Canis lupus familiaris
C1A1 Xenopus laevis
C1A2 Homo sapiens
C1A2 Danio rerio
C1A2 Mus musculus
C1A2 Rattus norvegicus
C1A2 Bos taurus
C1A2 Canis lupus familiaris
C1A2 Gallus gallus
C1A3 Danio rerio
C2A1 Bos taurus
C2A1 Homo sapiens
C2A1 Mus musculus
C2A1 Rattus norvegicus
C2A1 Canis lupus familiaris
C2A1 Gallus gallus
C2A1 Xenopus laevis
C2A1 Equus caballus
C2A1 Xenopus tropicalis
C3A1 Homo sapiens
C3A1 Mus musculus
C3A1 Rattus norvegicus
C3A1 Bos taurus
C3A1 Gallus gallus

AGAPGKDGLNGLPGPIGPPGPRGRTGDAGPVGPPGPPGPPGPPGPPSAGFDF AGSAGKDGMSGLPGPIGPPGPRGRNGEIGPAGPPGPPGPPGAPGPSGGGEDI AGSPGKDGLNGLPGPIGPPGPRGRTGDSGPAGPPGPPGPPGPPGPPSGGYDE AGSPGKDGLNGLPGPIGPPGPRGRTGDSGPAGPPGPPGPPGPPGPPSGGYDF AGSPGKDGLNGLPGPIGPPGPRGRTGDAGPAGPPGPPGPPGPPGPPSGGYDF AGVPGKDGMSGLPGPTGPPGPRGRSGEMGPAGPPGPPGPAGAPGAPGGGEDL AGSPGKDGLNGLPGPIGPPGPRGRTGDAGPVGPPGPPGPPGPPGPPSGGEDF SGNPGKDGSNGLPGPIGPPGPRGRTGDVGPAGPPGPPGPPGPPGQSGGGEDE SGPAGKDGRTGHPGTVGPAGIRGPQGHQGPAGPPGPPGPPGPPGVSGGGYDE NGPAGKDGSNGMPGAIGPPGHRGPAGHVGPAGPPGSPGLPGPPGPSGGGYDT SGPVGKDGRSGQPGPVGPAGVRGSQGSQGPAGPPGPPGPPGPPGVSGGGYDE SGPIGKDGRSGHPGPVGPAGVRGSQGSQGPAGPPGPPGPPGPPGVSGGGYDE SGPAGKDGRIGQPGAVGPAGIRGSQGSQGPAGPPGPPGPPGPPGPSGGGYEF SGPAGKDGRTGQPGTVGPAGIRGSQGSQGPAGPPGPPGPPGPPGPSGGGYDF SGPPGKDGRNGLPGPIGPAGVRGSHGSQGPAGPPGPPGPPGPPGPNGGGYEV NGSPGKDGMNGVPGPVGPPGPRGRSGEMGPAGAPGLPGPPGPPGSAGPGEPE VGPSGKDGANGIPGPIGPPGPRGRSGETGPAGPPGNPGPPGPPGPPGPGIDM VGPSGKDGANGIPGPIGPPGPRGRSGETGPAGPPGNPGPPGPPGPPGPGIDM VGPSGKDGSNGIPGPIGPPGPRGRSGETGPVGPPGSPGPPGPPGPPGPGIDM VGPSGKDGSNGIPGPIGPPGPRGRSGETGPAGPPGNPGPPGPPGPPGPGIDM VGPSGKDGANGIPGPIGPPGPRGRSGETGPAGPPGNPGPPGPPGPPGPGIDM VGPSGKDGSNGMPGPIGPPGPRGRSGEPGPAGPPGNPGPPGPPGPPGTGIDM VGPSGKDGSNGIPGPIGPPGPRGRSGETGPAGPPGQSGPPGPPGPPGPGIDM VGPSGKDGANGIPGPIGPPGPRGRSGETGPAGPPGNPGPPGPPGPPGPGIDM VGPSGKDGSNGLPGPIGPPGPRGRGGETGPAGPPGQPGPPGPPGPPGPGIDM SGPPGKDGTSGHPGPIGPPGPRGNRGERGSEGSPGHPGQPGPPGPPGAPGPC HGPPGKDGTSGHPGPIGPPGPRGNRGERGSEGSPGHPGQPGPPGPPGAPGPC HGPPGKDGSSGHPGPIGPPGPRGNRGERGSEGSPGHPGQPGPPGPPGAPGPC SGPPGKDGASGHPGPIGPPGPRGNRGERGSEGSPGHPGQPGPPGPPGAPGPC AGPPGKDGRGGYPGPIGPPGPRGNRGESGPAGPPGQPGLPGPSGPP---GPC

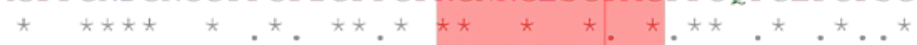

Imino poor region

Figure S6. Multiple sequence alignment of the last 52 residues of the C-terminal region of the triple helical domain of fibrillar collagen. The imino poor region near the C-terminus is shown with an orange background. 